Article

\title{
Investigation of Grignard Reagent as an Advanced Base for Aza-Claisen Rearrangement
}

\author{
Bo Reum Song ${ }^{1}$, Min Woo Ha ${ }^{1}$, Donghwan Kim ${ }^{2}$, Chanin Park ${ }^{2}$, Keun Woo Lee ${ }^{2}$ and \\ Seung-Mann Paek ${ }^{1, *}$ \\ 1 College of Pharmacy, Research Institute of Pharmaceutical Sciences, Gyeongsang National University, \\ Jinju daero, Jinju, Gyeongnam 52828, Korea; qhfma2005@naver.com (B.R.S.); \\ beneminu_@naver.com (M.W.H.) \\ 2 Division of Life Science, Division of Applied Life Science (BK21 Plus), Research Institute of Natural Science, \\ Gyeongsang National University, Jinju 52828, Korea; donghwan0651@gmail.com (D.K.); \\ chaninpark0806@gmail.com (C.P.); kwlee@gnu.ac.kr (K.W.L.) \\ * Correspondence: million@gnu.ac.kr; Tel.: +82-55-772-2424
}

Academic Editor: Alessandro Palmieri

Received: 7 November 2019; Accepted: 12 December 2019; Published: 16 December 2019

check for updates

\begin{abstract}
Employing $i \mathrm{PrMgCl}$ as an advanced base instead of lithium hexamethyldisilazane (LHMDS) resulted in dramatic improvements in aza-Claisen rearrangement. This advance is considered responsible for the increased bulkiness of the alkoxide moiety (including magnesium cation and ligands), followed by a resultant conformational change of the transition state. To support this hypothesis, various substrates of aza-Claisen rearrangement were prepared and screened. In addition, a molecular dynamic simulation study was performed to investigate and compare the structural stability of reaction intermediates.
\end{abstract}

Keywords: aza-Claisen rearrangement; Grignard reagent; ring expansion; base; molecular dynamics

\section{Introduction}

Azacycles, nitrogen containing cyclic molecules, has important biological activity and synthetic utility [1]. Various conversions of azacycle skeletons have contributed to the construction of alkaloid frameworks and the development of important synthetic methodologies (the aza-Cope rearrangement [2], transannulation [3], Diels-Alder cycloaddition [4], and so on [5]). However, their synthetic applications require further development to improve chemical yields, handling, and substrate generality. Aza-Claisen rearrangement (ACR) is one of these methodologies [6]. The [3,3]-sigmatropic rearrangement of nitrogen containing a diene moiety serves as a robust platform to introduce various alkaloid skeletons into natural products or active pharmaceutical ingredients (APIs) [7]. More importantly, with ACR employed, chiral communication and induction of remote stereogenic centers were also reported in a highly selective manner [8,9] (Figure 1). However, this type of rearrangement also requires harsh reaction conditions and at times results in low yields [10].

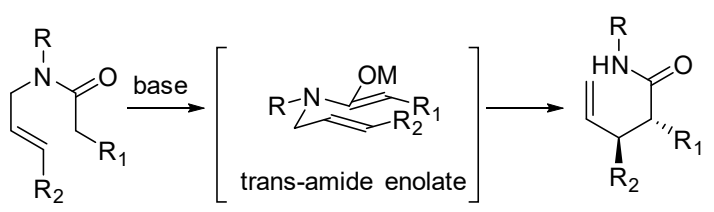

Figure 1. Aza-Claisen rearrangement (ACR) through amide enolate and its stereochemical outcome.

ACR proceeds through deprotonation, [3,3]-sigmatropic rearrangement, and protonation, as shown in Figure 2. For this process, both a strong base for deprotonation of $\alpha$-hydrogen in an amide 
group and a high temperature for thermal rearrangement are required [11,12]. However, this reaction condition also permits sigma-bond rotation in amide enolate to hamper generation of the desired conformation. If the undesired conformation exists as a major form, ACR would be impossible (low conversion), and an ensuing side reaction would occur (side product). Because the oxygen-metal bond may play an important role in this enolate conformation, it can be presumed that the cation of a base determines a successful ACR process. Actually, it was shown that lithium hexamethyldisilazane (LHMDS) and $i \mathrm{PrMgCl}$ gave different results in the ACR of an API synthesis [13].

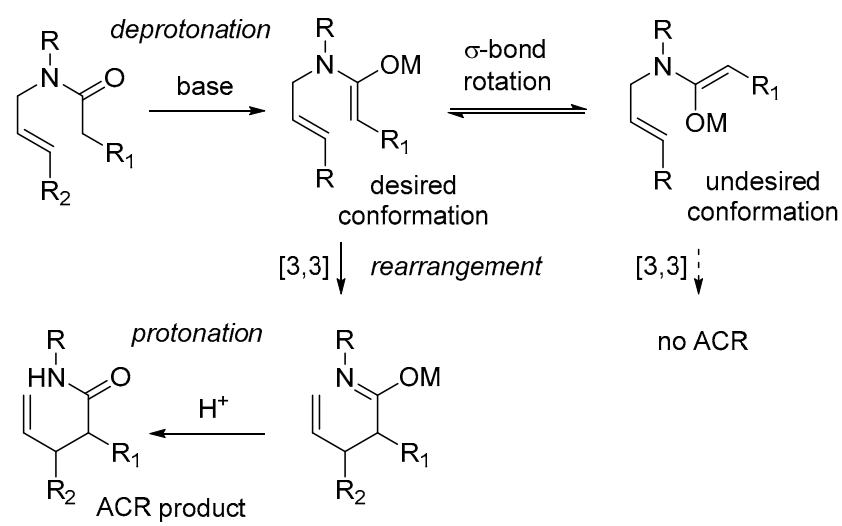

Figure 2. Equilibrium of an amide enolate conformation.

Inspired by this base-dependent ACR, we tried to apply this methodology to a more general substrate. It was expected that controlling an amide enolate conformation's equilibrium through various enolate moieties, including metal cations and ligands, would mitigate the undesired side reaction in accelerated ACR processes. Based on this hypothesis, a simple ACR substrate was designed to screen bases.

\section{Results}

To prove the efficiency of the cation-dependent ACR, large amounts of substrate were pursued in a short time. In addition, cyclic ACR substrate was favored over acyclic because the ACR of cyclic substrates proceeds with fewer entropic/geometric issues [12]. Commercially available methyl pipecolinate $\mathbf{1}$ was subjected to the sequential Schotten-Baumann amidation [14] to create amide 2, which was converted to aldehyde 3 via diisobuylaluminum hydride (DIBAL) reduction. Finally, Wittig methylenation afforded allyl amide 4 an excellent yield, as depicted in Scheme 1.

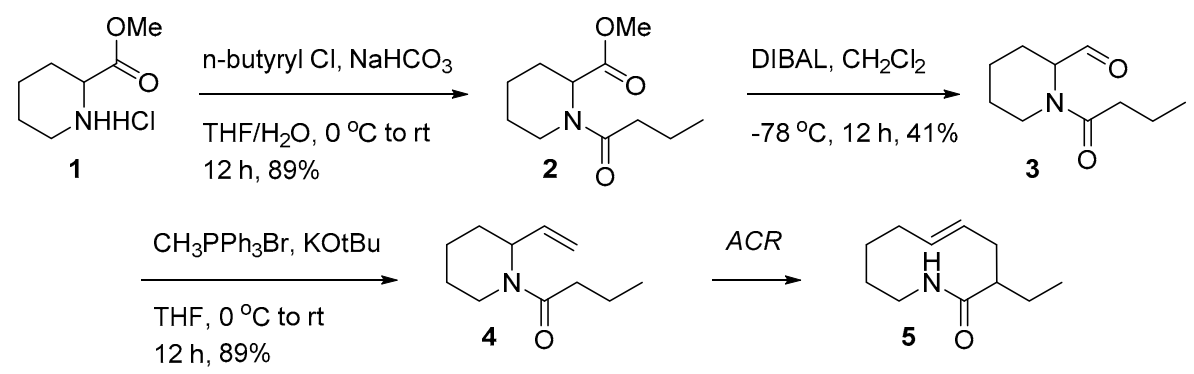

Scheme 1. Preparation of substrate for ACR. DIBAL: diisobuylaluminum hydride; THF: tetrahydrofuran.

Various conditions were screened to effectively convert allyl amide 4 to the ring-expanded lactam 5, as shown in Table 1. As expected, bulky Grignard reagents, such as $i \mathrm{PrMgCl}$ or $t \mathrm{BuMgBr}$, exhibited greater efficiency than LHMDS, the standard base for ACR [15,16] (entries 1, 2, 4). In contrast, $s p^{2}$-hybridized carbanion, such as 2-mesitylMgBr, produced a lower yield than LHMDS [17] (entry 3). Relatively small Grignard reagents were also not effective, likely because of the nucleophilic 
substitution of Grignard reagents. Although sodium and potassium cations are larger than lithium cations, employing NaHMDS or KHMDS as a base also generated worse results than LHMDS (entries 7,8 ): in these cases, rather than the ACR adduct 5 , an unidentified side product was obtained soon after the base was added. By contrast, $i \mathrm{PrMgCl}$ improved the ACR process, but other reaction conditions, such as the solvent and temperature, needed to be further optimized. The screening of representative solvents (benzene, tetrahydrofuran (THF), $n$-decane) is depicted in entries 9-11. The use of benzene improved results slightly; however, polar or extremely nonpolar solvents did not. Lastly, an ACR carried out at room temperature with benzene yielded no reaction (entry 12). Replacing $i \mathrm{PrMgCl}$ with $i \mathrm{PrMgBr}$ showed similar results, as shown in entry 13.

Table 1. Screening of ACR conditions. LHMDS: lithium hexamethyldisilazane.

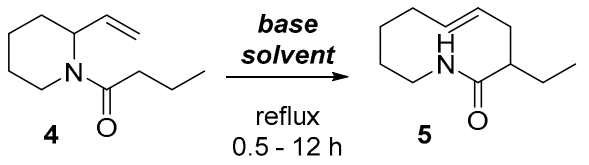

\begin{tabular}{cccc}
\hline Entry & Base & Solvent & Result (\%) $^{\mathbf{a}}$ \\
\hline 1 & LHMDS & toluene & 62 \\
2 & $i$ PrMgCl & toluene & 78 \\
3 & 2-MesitylMgBr & toluene & 53 \\
4 & t-BuMgBr & toluene & 64 \\
5 & $n$-PropylMgBr & toluene & 21 \\
6 & EtMgBr & toluene & 31 \\
7 & NaHMDS & toluene & $-\mathrm{b}$ \\
8 & KHMDS & toluene & $-\mathrm{b}$ \\
9 & $i$ PrMgCl & benzene & 84 \\
10 & $i$ PrMgCl & THF & 23 \\
11 & $i P r M g C l$ & $n$-decane & 43 \\
12 & $i$ PrMgCl & benzene & c \\
13 & $i$ PrMgCl & benzene & 81 \\
\hline
\end{tabular}

a Isolation yield; ${ }^{\mathrm{b}}$ Unidentified side product was obtained in $1 \mathrm{~min} . ;^{\mathrm{c}}$ Reaction was carried out at the room temperature.

The optimized ACR conditions were applied to various substrates, as shown in Scheme 2. The reduction of known ester 6 [18] provided primary alcohol 7, which was protected by a tert-butyldiphenylsilyl (TBDPS) group. The silyl ether 8 was treated with trifluoroacetic acid (TFA) to generate amine salt, which was transformed uneventfully into butyryl amide 9. To verify the bulkiness of allyl side chain 9, TBDPS was converted into tert-butyldimethylsilyl (TBS) or triethylsilyl (TES) through tetra- $n$-butylammonium fluoride (TBAF)/AcOH deprotection followed by a corresponding silyl protection. This straightforward synthesis enabled TBS or TES to substitute for ACR substrates $\mathbf{1 1}$ and $\mathbf{1 2}$ in an excellent yield. Amide substitution was also attempted. After acidic deprotection of the tert-butyloxycarbonyl (Boc) group of carbamates $\mathbf{8}$, as described above, a concomitant acylation with alkynyl or aromatic functionality was also executed, employing $N$-ethyl- $N^{\prime}$-(3-dimethylaminopropyl)carbodiimide hydrochloride(EDCI)/4-dimethylaminopyridine (DMAP) amidation conditions. After two uneventful procedures, desired substrates $\mathbf{1 3}$ and $\mathbf{1 4}$ were produced. 

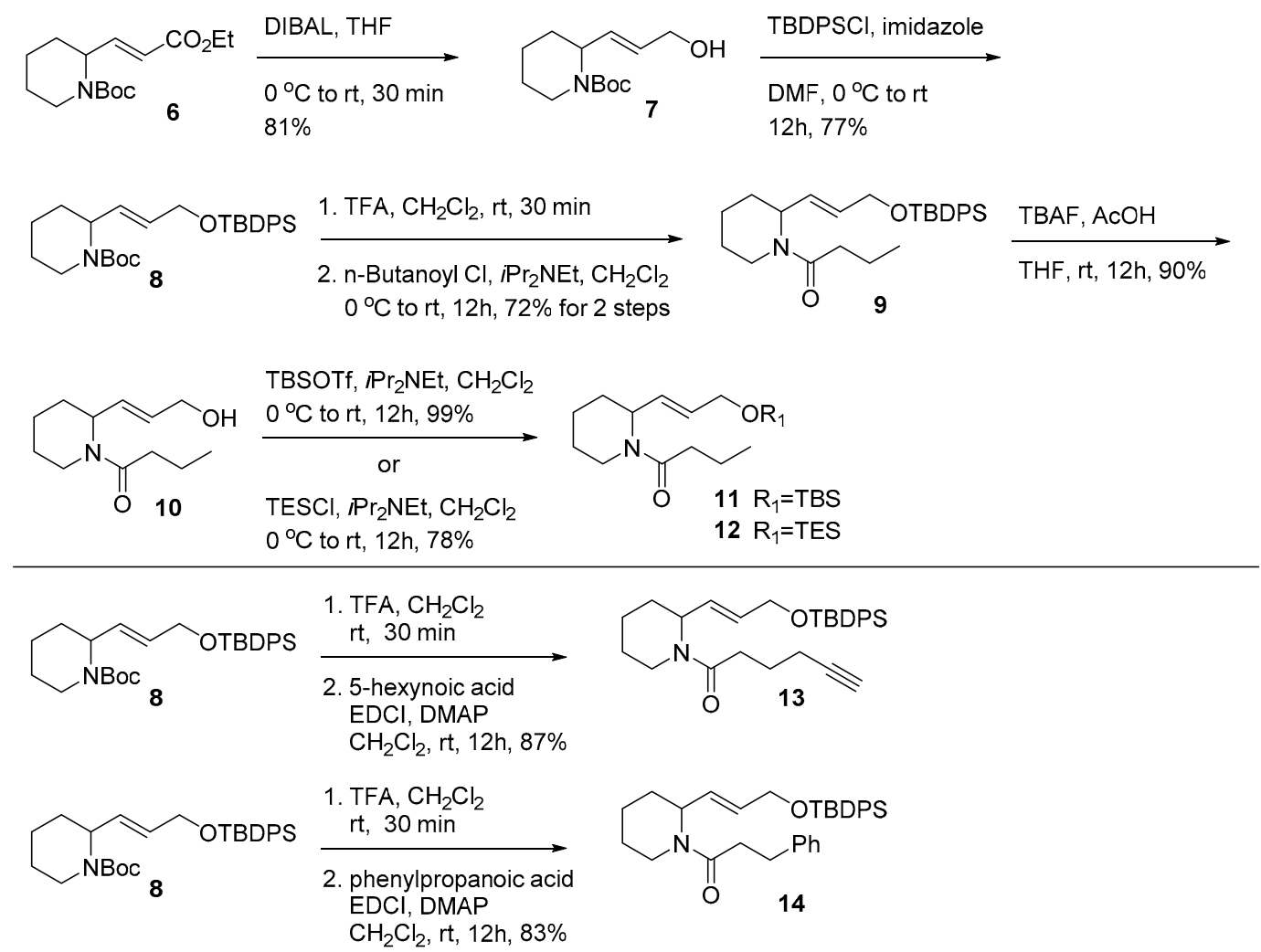

Scheme 2. Preparation of various substrates in alkene moiety. TFA: trifluoroacetic acid; TBAF: tetra- $n$-butylammonium fluoride; EDCI: $N^{\prime}$-(3-dimethylaminopropyl)carbodiimide hydrochloride; DMAP: 4-dimethylaminopyridine; TBS: tert-butyldimethylsilyl.

Amide substituent derivatization was also carried out, as shown in Scheme 3. Williamson etherification of the primary alcohol 7, followed by sequential Boc deprotection/amidation, afforded differently substituted amides $\mathbf{1 6 - 1 8}$ in a straightforward manner. These three derivatives were expected to prove the substituent effect of amide moiety.

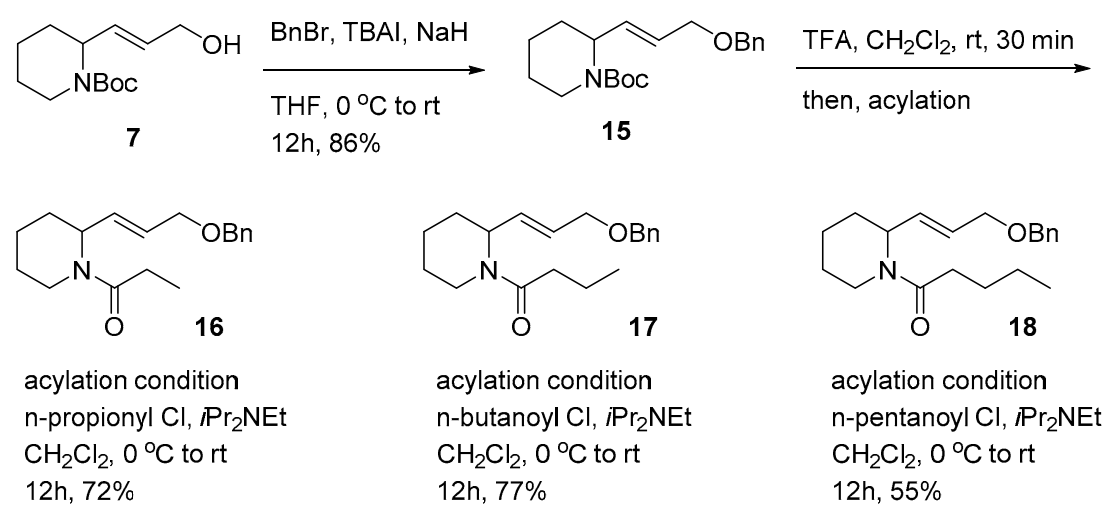

Scheme 3. Preparation of various ACR substrates in amide moiety. TBAI: tetra- $n$-butylammonium iodide.

Finally, acyclic substrate 21 was prepared from $p$-methoxybenzyl amines 19 through an amidation/crotylation sequence. Thus, with the various substrates in hand, $i \mathrm{PrMgCl}$ mediated ACR (Scheme 4). 


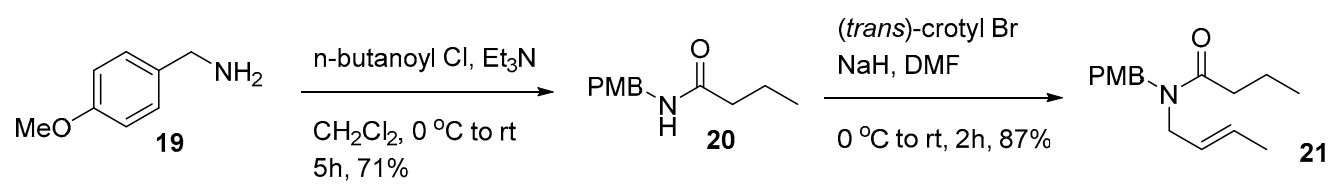

Scheme 4. Preparation of acyclic ACR substrates. DMF: tetra- $n$-butylammonium fluoride; PMB: para-nethoxyl benzyl.

The ACR of various substrates is summarized in Scheme 5. As expected, the employment of $i \mathrm{PrMgCl} /$ benzene converted each of the substrates into the requisite lactams/amides more efficiently than in conventional conditions (LHMDS/toluene). Of note, superior results occurred in some cases, such as sterically demanded substrates 13 and 14, as well as labile TES-protected substrate 12. ACR under the treatment of acyclic amide $\mathbf{2 1}$ with $i \mathrm{PrMgCl}$ also yielded impressive results. Moreover, the LHMDS base did not produce the desired free amide $2 \mathbf{1}^{\prime}$ at all. In fact, a brief comparison of the alkene-substituted ACR substrate showed that the larger the substituent of the allylic side chain, the worse the product yield. Based on this tendency, the increased bulkiness of the side chain could cause the desired ACR process to occur. Similarly, the results from amide analogs 16-18 also supported this hypothesis. Consequently, increased repulsion of the allylic side chain with amide enolate is thought to be responsible for the low yield of ACR, but this obstacle can be overcome by employing $i \mathrm{PrMgCl}$ as a base.

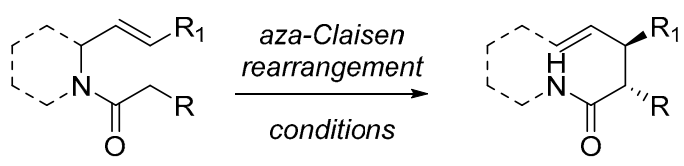

condition A

2eq. $\mathrm{PrMgCl}$, benzene, reflux, $0.5-17 \mathrm{~h}$

condition B

2eq. LHMDS, toluene, reflux, 5-15h
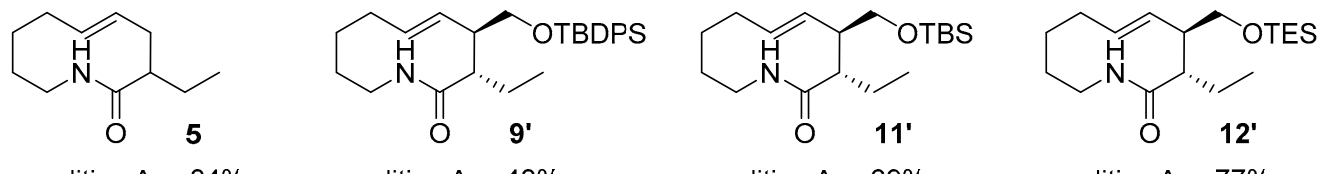

condition A $84 \%$

condition A $48 \%$ condition B $62 \%$ condition B $\quad 7.5 \%$

condition A $\quad 69 \%$ condition B $25 \%$

condition A $\quad 77 \%$ condition B not dectected

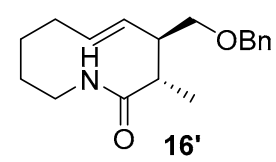

condition A $80 \%$ condition B $35 \%$

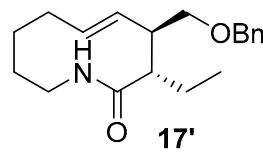

condition A $70 \%$ condition B $15 \%$

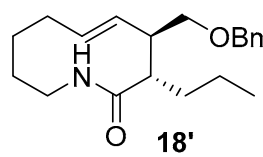

condition A $\quad 66 \%$ condition B not dectected

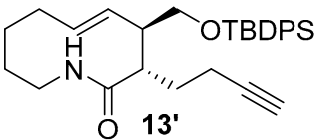

condition A $55 \%$ condition $B$ not dectected

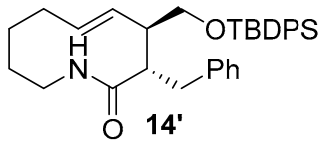

condition $\mathrm{A}$ ( 5 eq. base) $94 \%$ condition B not dectected

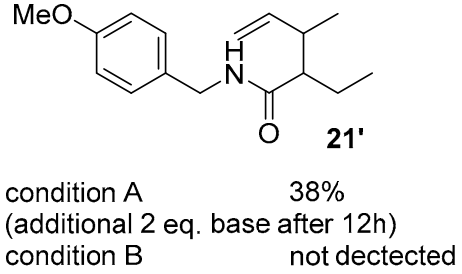

Scheme 5. Comparison of ACR conditions using various substrates.

To verify the structure of macrolactams, ACR product 14' was crystallized. Figure 3 represents the structure of $14^{\prime}$, which features the 1,2-anti-configured side chain and the 10-membered lactam framework. The crystal structure below shows that ACR proceeded as designed. 


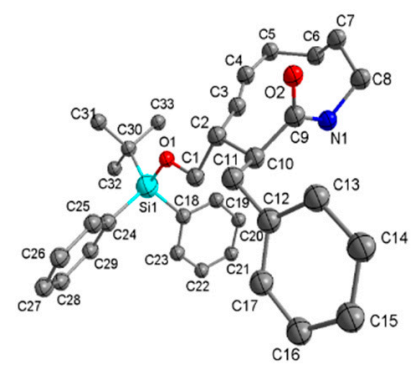

Figure 3. Crystal structure of macrolactam 14'.

From a mechanistic point of view, it was hypothesized that reactive conformation would explain the pivotal role of $i \mathrm{PrMgCl}$. For example, in the case of amide 17, a reactive enolate had to be generated and arranged for the desired [3,3]-sigmatropic rearrangement to occur. While desired conformer 17-A might produce a corresponding ACR product, the undesired conformer 17-B might lead to no ACR process. The portion of the undesired conformer 17-B that resulted from the substrate $\mathbf{1 7}$ could be lessened when the Grignard base was used instead of LHMDS, as steric repulsion of the alkenyl side chain with Cl-Mg-O complex (in 17-D) made it more unstable than the Li-O complex did (in 17-B). This repulsion might give rise to the rapid conversion of the undesired conformer 17-D to the desired conformer 17-C, which would result in the ACR process (Scheme 6).

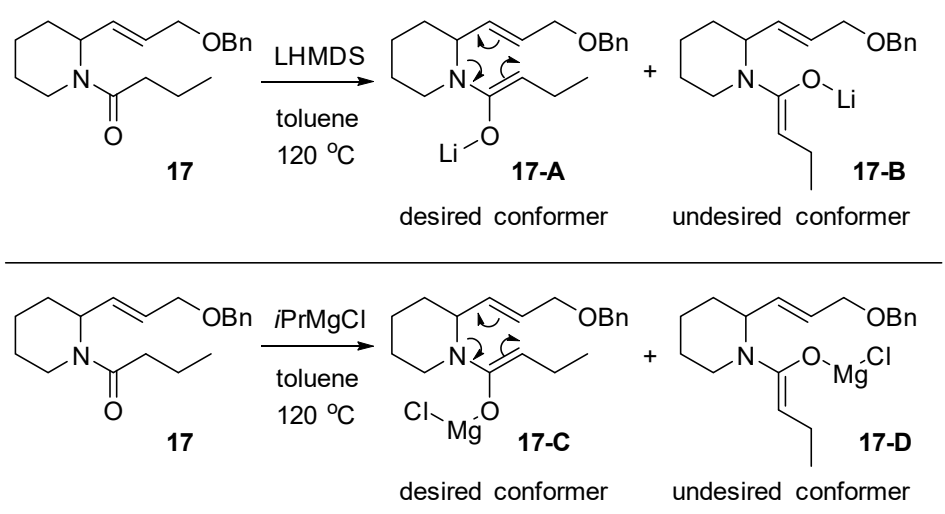

Scheme 6. Mechanistic view of cation-dependent ACR.

To investigate the structural stability of the reaction intermediates and their conformations (17-A through 17-D), molecular dynamics (MD) simulations were carried out for $10 \mathrm{~ns}$. To monitor the structural stability of each conformation, the C-C-C-O dihedral angles were measured during the simulations (Figure 4). 
A<smiles>[R]/C(=C\CC)N1CCCCC1/C=C/COc1ccccc1</smiles><smiles>[R]/C(=C/CC)N1CCCCC1/C=C/COCc1ccccc1</smiles>

B
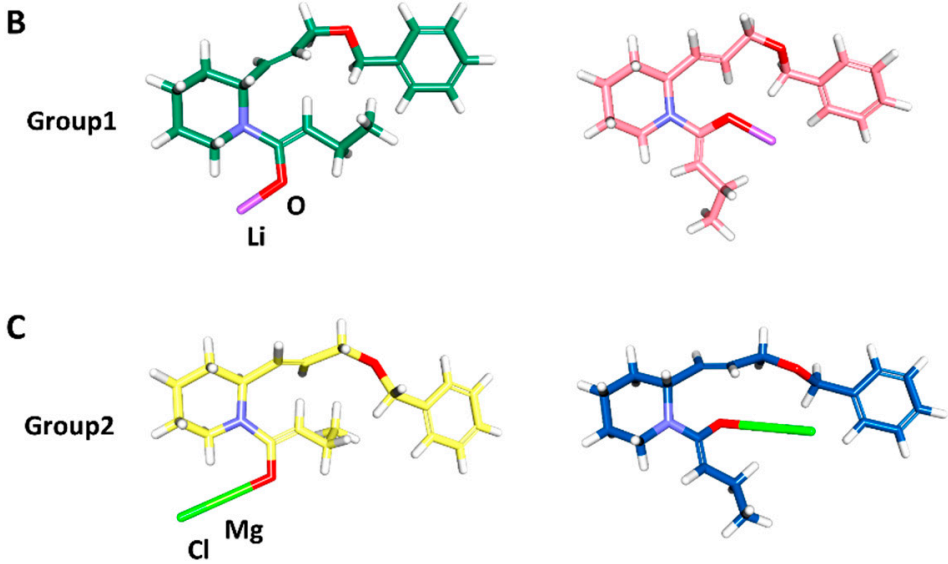

Figure 4. 2D and 3D structures of four different conformations of 19 compound. (A) Sketch of trans, cis conformations and C-C-C-O dihedral angle. 3D structure of each compound in (B) Group1 and (C) Gruop2.

In the case of lithium enolate (Figure 5), MD results clearly showed that the desired Li-conformer 17-A is very unstable: it did not stay in one state for long (Figure 5A,B). In contrast, the conformation of 17-B was stable, maintaining the C-C-C-O dihedral angle at $60^{\circ}$ (Figure 5A,C).

A

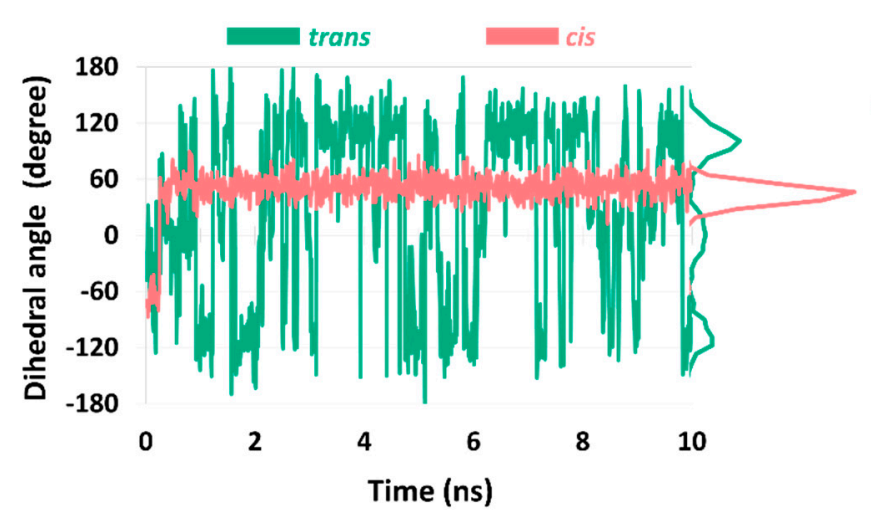

C

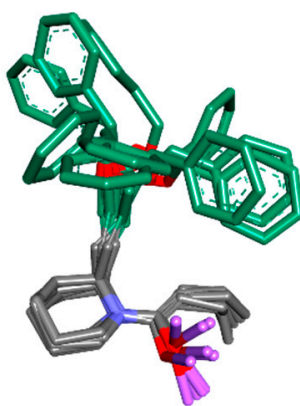

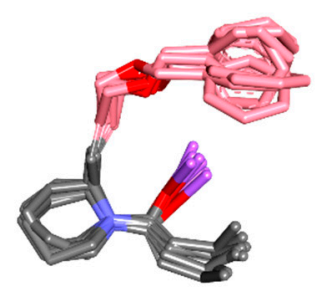

Figure 5. Comparison of structural stability of trans and cis conformations of Group1 compound. (A) Dihedral angle in CCCO of trans and cis conformations. Cartoon of structure superposition of every nano second frames of cluster obtained from (B) trans (green) and (C) cis conformations (pink).

However, in the case of magnesium enolate from amide 17, the structural stability of both conformations showed similar fluctuation patterns (Figure 6A). The most common dihedral angles (i.e., conformation) of $16-\mathrm{C}$ were $110^{\circ}$ (Figure 6A,B), while those of 17-D were $-110^{\circ}$ (Figure 6A,C). The instability of undesired conformer 17-D would explain the rapid conversion of desired conformer 17-C in the corresponding ACR product. 
A

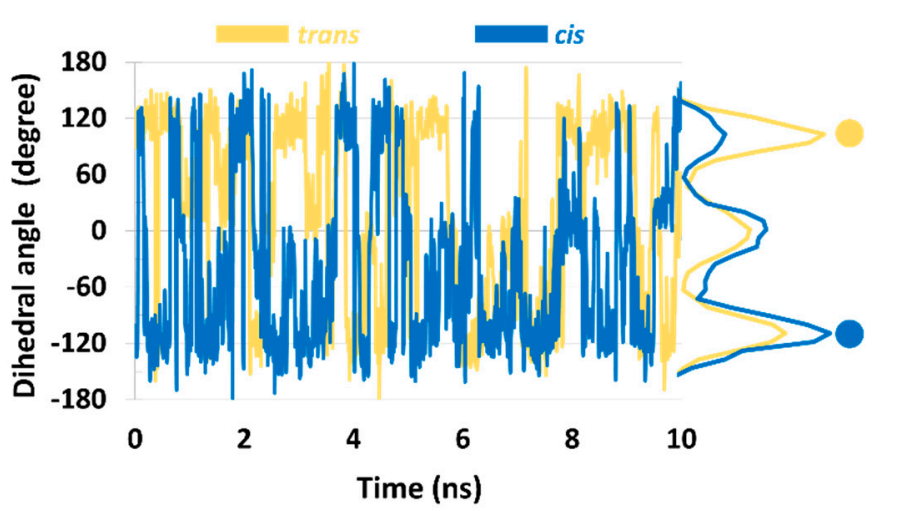

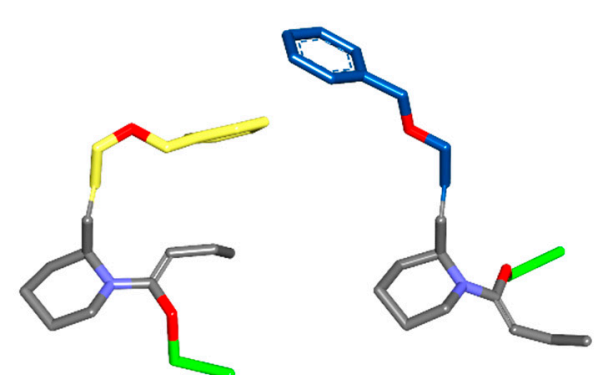

Figure 6. Comparison of structural stability of trans and cis conformations in Group2 compound. (A) Dihedral angle in CCCO of trans and cis conformations. Cartoon of structure of the most popular clusters obtained from (B) trans (yellow) and (C) cis conformations (blue).

\section{Materials and Methods}

\subsection{General Information}

Unless noted otherwise, all starting materials and reagents were obtained from commercial suppliers and were used without further purification. Tetrahydrofuran was distilled from sodium benzophenone ketyl. Dichloromethane was freshly distilled from calcium hydride. All solvents used for routine isolation of products and chromatography were reagent grade. Air- and moisture-sensitive reactions were performed under argon atmosphere. Flash column chromatography was performed using silica gel 60 (230-400 mesh, Merck, Darmstadt, Germany) with the indicated solvents. Thin-layer chromatography was performed using $0.25 \mathrm{~mm}$ silica gel plates (Merck). ${ }^{1} \mathrm{H}-\mathrm{NMR}$ data were reported in the order of chemical shift, multiplicity (s, singlet; $d$, doublet; $t$, triplet; $q$, quartet; $m$, multiplet and/or multiple resonance), number of protons, and coupling constant in hertz ( $\mathrm{Hz}$ ).

\subsubsection{Methyl 1-butyrylpiperidine-2-carboxylate (2)}

To a solution of methyl pipecolinate hydrochloride $1(3.9 \mathrm{~g}, 22 \mathrm{mmol})$ in THF $(40 \mathrm{~mL})$, a solution of $\mathrm{Na}_{2} \mathrm{CO}_{3}(11 \mathrm{~g}, 110 \mathrm{mmol})$ in $\mathrm{H}_{2} \mathrm{O}$ was added at $0{ }^{\circ} \mathrm{C}$. After addition of n-butyryl chloride $(2.7 \mathrm{~mL}$, $26 \mathrm{mmol}$ ) at $0{ }^{\circ} \mathrm{C}$, the reaction mixture was stirred for $24 \mathrm{~h}$. After filtration of insoluble solids, reaction mixture was extracted EtOAc twice. Organic layers were dried over $\mathrm{MgSO}_{4}$, filtered, evaporated, and purified by column chromatography on silica gel (EtOAc:n-hexane $=1: 1$ ) to afford methyl ester 2 $(4.1 \mathrm{~g}, 89 \%)$ as a colorless oil. IR (KBr) $v_{\max } 2953,2869,1741,1648,1426,1322 \mathrm{~cm}^{-1} ;{ }^{1} \mathrm{H}-\mathrm{NMR}\left(\mathrm{CDCl}_{3}\right.$, $400 \mathrm{MHz}$, mixture of rotamers) $\delta 5.25(\mathrm{~d}, 1 \mathrm{H}, J=5.2 \mathrm{~Hz}), 4.47-4.40,3.65-3.61(\mathrm{~m}, 1 \mathrm{H}), 3.57(\mathrm{~s}, 3 \mathrm{H})$, $3.09(\mathrm{dt}, 1 \mathrm{H}, J=2.8,12.8 \mathrm{~Hz}), 2.48-2.47,2.18-2.03(\mathrm{~m}, 2 \mathrm{H}), 2.24(\mathrm{t}, 2 \mathrm{H}, J=7.6 \mathrm{~Hz}), 1.59-1.43(\mathrm{~m}, 4 \mathrm{H})$, $1.36-1.13(\mathrm{~m}, 2 \mathrm{H}), 0.83(\mathrm{t}, 3 \mathrm{H}, J=7.6 \mathrm{~Hz}) ;{ }^{13} \mathrm{C}-\mathrm{NMR}\left(\mathrm{CDCl}_{3}, 100 \mathrm{MHz}\right.$, mixture of rotamers) $\delta 172.7$, 172.3, 171.6, 171.1, 55.7, 52.0, 51.7, 51.4, 43.1, 38.9, 35.0, 34.7, 27.0, 26.3, 25.0, 24.3, 20.7, 20.6, 18.2, 13.5; LRMS (FAB) $m / z 214\left(\mathrm{M}+\mathrm{H}^{+}\right)$; HRMS (FAB) calcd for $\mathrm{C}_{11} \mathrm{H}_{20} \mathrm{NO}_{3}\left(\mathrm{M}+\mathrm{H}^{+}\right)$: 214.1443, found 214.1448.

\subsubsection{1-Butyrylpiperidine-2-carbaldehyde (3)}

To a solution of methyl ester $2(850 \mathrm{mg}, 4.0 \mathrm{mmol})$ in $\mathrm{CH}_{2} \mathrm{Cl}_{2}(10 \mathrm{~mL})$, DIBAL (1.0 M in toluene, $8.0 \mathrm{~mL}, 8.0 \mathrm{mmol}$ ) was added at $-78{ }^{\circ} \mathrm{C}$ and stirred for $3 \mathrm{~h}$. Then, $15 \%$ sodium potassium tartrate solution $(10 \mathrm{~mL})$ was added to reaction mixture and stirred for $12 \mathrm{~h}$ at room temperature. Reaction mixture was extracted $\mathrm{CH}_{2} \mathrm{Cl}_{2}$ twice. Organic layers were dried over $\mathrm{MgSO}_{4}$, filtered, evaporated, and purified by column chromatography on silica gel (EtOAc: $n$-hexane $=1: 1$ ) to afford aldehyde 3 (300 mg, 41\%) as a colorless oil. IR (KBr) $v_{\max } 2939,2869,1731,1643,1425 \mathrm{~cm}^{-1} ;{ }^{1} \mathrm{H}-\mathrm{NMR}\left(\mathrm{CDCl}_{3}\right.$, $400 \mathrm{MHz}$, mixture of rotamers) $\delta 9.62(\mathrm{~s}), 9.45(\mathrm{~s}, 1 \mathrm{H}), 5.33(\mathrm{~d}, J=5.2 \mathrm{~Hz}), 5.07-5.05(\mathrm{~m}, 1 \mathrm{H}), 4.62-4.58$ 
(m), 4.35-4.33 (m), 3.72-3.64 (m, 2H), $3.08(\mathrm{dt}, 1 \mathrm{H}, J=2.8,11.6 \mathrm{~Hz}), 2.35-2.13(\mathrm{~m}, 4 \mathrm{H}), 1.68-1.53(\mathrm{~m}, 5 \mathrm{H})$, $1.43-1.18(\mathrm{~m}, 2 \mathrm{H}), 0.89(\mathrm{t}, 3 \mathrm{H}, J=7.2 \mathrm{~Hz}) ;{ }^{13} \mathrm{C}-\mathrm{NMR}\left(\mathrm{CDCl}_{3}, 100 \mathrm{MHz}\right.$, mixture of rotamers) $\delta 201.0$, 200.2, 173.1, 62.3, 58.9, 51.9, 51.6, 44.1, 43.3, 35.2, 35.0, 26.5, 25.3, 24.5, 23.2, 20.9, 20.8, 18.5, 13.7; LRMS (FAB) $m / z 184\left(\mathrm{M}+\mathrm{H}^{+}\right)$; HRMS (FAB) calcd for $\mathrm{C}_{10} \mathrm{H}_{18} \mathrm{NO}_{2}\left(\mathrm{M}+\mathrm{H}^{+}\right)$: 184.1338, found 184.1340.

\subsubsection{1-(2-Vinylpiperidin-1-yl)butan-1-one (4)}

To a suspension of methyl triphenylphosphonium bromide (850 mg, $2.4 \mathrm{mmol})$ in THF (5 mL), $\mathrm{KOtBu}(1.0 \mathrm{M}$ in THF, $2.2 \mathrm{~mL}, 2.2 \mathrm{mmol})$ was added at $0{ }^{\circ} \mathrm{C}$ and stirred for $30 \mathrm{~min}$. After addition of aldehyde 2 (290 mg, $1.6 \mathrm{mmol})$ in THF ( $2 \mathrm{~mL})$, reaction mixture was stirred for $10 \mathrm{~min}$ and quenched with addition of $\mathrm{H}_{2} \mathrm{O}$. Reaction mixture was extracted EtOAc twice. Organic layers were dried over $\mathrm{MgSO}_{4}$, filtered, evaporated and purified by column chromatography on silica gel (EtOAc: $n$-hexane $=1: 2$ to $1: 1)$ to afford allyl amide $4(220 \mathrm{mg}, 77 \%)$ as a colorless oil. IR (KBr) $v_{\max } 2937,2867,1645,1425$, $1244 \mathrm{~cm}^{-1} ;{ }^{1} \mathrm{H}-\mathrm{NMR}\left(\mathrm{CDCl}_{3}, 400 \mathrm{MHz}\right.$, mixture of rotamers) $\delta 5.71-5.68(\mathrm{~m}, 1 \mathrm{H}), 5.17(\mathrm{~m}, 1 \mathrm{H}), 5.34$ (bs), 3.61-3.58 (m, 1H), 5.02-4.98 (m, 1H), $4.48(\mathrm{bs}, 1 \mathrm{H}), 3.08(\mathrm{t}, J=11.6 \mathrm{~Hz}), 2.60(\mathrm{t}, 1 \mathrm{H}, J=11.6 \mathrm{~Hz})$, 2.32-2.20 (m, 2H), 1.79-1.76 (m, 1H), 1.66-1.40 (m, 6H), $1.34(\mathrm{~m}, 1 \mathrm{H}), 0.93-0.89(\mathrm{~m}, 3 \mathrm{H}) ;{ }^{13} \mathrm{C}-\mathrm{NMR}$ $\left(\mathrm{CDCl}_{3}, 100 \mathrm{M} \mathrm{Hz}\right) \delta 172.4,171.7,153.5,136.5,116.2,115.8,54.4,51.6,49.5,43.3,41.7,37.2,35.5,35.3,35.0$, 30.0, 28.3, 26.3, 25.2, 19.5, 18.8, 13.9; LRMS (FAB) $m / z 182\left(\mathrm{M}+\mathrm{H}^{+}\right)$; HRMS (FAB) calcd for $\mathrm{C}_{11} \mathrm{H}_{20} \mathrm{NO}$ $\left(\mathrm{M}+\mathrm{H}^{+}\right):$182.1545, found 182.1543 .

\subsection{4. (E)-3-ethyl-3,4,7,8,9,10-hexahydroazecin-2(1H)-one (5)}

Procedure A; To a solution of allyl amide $4(29 \mathrm{mg}, 0.15 \mathrm{mmol})$ in toluene $(1 \mathrm{~mL}), i \operatorname{PrMgCl}(2.0 \mathrm{M}$ in THF, $0.15 \mathrm{~mL}, 0.30 \mathrm{mmol}$ ) was added at reflux condition. After stirring at same temperature for $30 \mathrm{~min}$, reaction mixture was cooled down to room temperature and quenched with brine and extracted with EtOAc. Organic layers were dried over $\mathrm{MgSO}_{4}$, filtered, evaporated and purified by column chromatography on silica gel (EtOAc: $n$-hexane $=2: 1)$ to afford lactam $5(22 \mathrm{mg}, 78 \%)$ as an amorphous solid.

Procedure B; To a solution of allyl amide $4(29 \mathrm{mg}, 0.15 \mathrm{mmol})$ in toluene $(2 \mathrm{~mL})$, LHMDS (1.0 M in n-hexane, $0.30 \mathrm{~mL}, 0.30 \mathrm{mmol}$ ) was added at reflux condition. After stirring at same temperature for $12 \mathrm{~h}$, reaction mixture was cooled down to room temperature and quenched with brine and extracted with EtOAc. Organic layers were dried over $\mathrm{MgSO}_{4}$, filtered, evaporated, and purified by column chromatography on silica gel (EtOAc: $n$-hexane $=2: 1)$ to afford lactam $5(18 \mathrm{mg}, 62 \%)$ as an amorphous solid. IR (KBr) $v_{\max } 3315,2925,2441,1637,1550,1451 \mathrm{~cm}^{-1} ;{ }^{1} \mathrm{H}-\mathrm{NMR}\left(\mathrm{CD}_{3} \mathrm{OD}, 300 \mathrm{M} \mathrm{Hz}\right) \delta 7.37$ (bs, $1 \mathrm{H}), 5.38-5.16(\mathrm{~m}, 2 \mathrm{H}), 3.49-3.41(\mathrm{~m}, 1 \mathrm{H}), 2.69-2.62(\mathrm{~m}, 1 \mathrm{H}), 2.17-2.10(\mathrm{~m}, 1 \mathrm{H}), 2.07-1.97(\mathrm{~m}, 2 \mathrm{H})$, 1.82-1.74 (m, 3H), 1.67-1.52 (m, 2H), 1.40-1.37 (m, 1H), 1.30-1.19 (m, 2H), $0.77(\mathrm{t}, 3 \mathrm{H}, \mathrm{J}=3.9 \mathrm{~Hz})$; ${ }^{13} \mathrm{C}-\mathrm{NMR}\left(\mathrm{CDCl}_{3}, 75 \mathrm{M} \mathrm{Hz}\right) \delta 174.8,134.5,127.7,52.7,40.2,37.3,32.8,29.7,29.0,24.1,12.4$; LRMS (FAB) $m / z 182\left(\mathrm{M}+\mathrm{H}^{+}\right)$; HRMS (FAB) calcd for $\mathrm{C}_{11} \mathrm{H}_{20} \mathrm{NO}\left(\mathrm{M}+\mathrm{H}^{+}\right): 182.1545$, found 182.1543.

\subsection{5. (E)-tert-butyl 2-(3-hydroxyprop-1-enyl)piperidine-1-carboxylate (7)}

To a solution of unsaturated ester $6(2.9 \mathrm{~g}, 10 \mathrm{mmol})$ in THF (20 mL), DIBAL (1.0 M in toluene, $22 \mathrm{~mL}, 22 \mathrm{mmol}$ ) was added at $0^{\circ} \mathrm{C}$ and stirred for $30 \mathrm{~min}$. $15 \%$ sodium potassium tartrate solution was added to reaction mixture and stirred for $5 \mathrm{~h}$ at room temperature. Reaction mixture was extracted EtOAc twice. Organic layers were dried over $\mathrm{MgSO}_{4}$, filtered, evaporated and purified by column chromatography on silica gel (EtOAc: $n$-hexane $=1: 1)$ to afford primary alcohol $7(2.0 \mathrm{~g}, 81 \%)$ as a colorless oil. IR (KBr) $v_{\max } 3443,2936,2862,1690,1515,1415,1367 \mathrm{~cm}^{-1} ;{ }^{1} \mathrm{H}-\mathrm{NMR}\left(\mathrm{CDCl}_{3}, 500 \mathrm{MHz}\right.$, mixture of rotamers) $\delta 5.64-5.56(\mathrm{~m}, 2 \mathrm{H}), 4.74(\mathrm{~b}, 1 \mathrm{H}) 5.58-5.54(\mathrm{~m}, 1 \mathrm{H}), 4.10-4.09(\mathrm{~m}, 2 \mathrm{H}), 3.88(\mathrm{~d}, 2 \mathrm{H}$, $J=13.5 \mathrm{~Hz}), 2.77(\mathrm{t}, 1 \mathrm{H}, J=12.5 \mathrm{~Hz}), 2.21(\mathrm{~b}, 1 \mathrm{H}), 1.72-1.58(\mathrm{~m}, 2 \mathrm{H}), 1.54-1.52(\mathrm{~m}, 2 \mathrm{H}), 1.40(\mathrm{~s}, 9 \mathrm{H})$; ${ }^{13} \mathrm{C}-\mathrm{NMR}\left(\mathrm{CDCl}_{3}, 100 \mathrm{MHz}\right.$, mixture of rotamers) $\delta 171.0,155.2,130.4,129.6,128.6,79.3,62.8,60.2,51.4$, 50.3, 39.6, 29.0, 28.3, 25.3, 20.8, 19.3, 18.9, 14.0; LRMS (FAB) $m / z 242\left(\mathrm{M}+\mathrm{H}^{+}\right)$; HRMS (FAB) calcd for $\mathrm{C}_{13} \mathrm{H}_{24} \mathrm{NO}_{3}\left(\mathrm{M}+\mathrm{H}^{+}\right): 242.1756$, found 242.1752 . 
3.1.6. (E)-tert-butyl 2-(3-(tert-butyldiphenylsilyloxy)prop-1-enyl)piperidine-1-carboxylate (8)

To a solution of alcohol 7 (920 mg, $3.8 \mathrm{mmol}$ ) in dimethylformamide (DMF, $8 \mathrm{~mL}$ ), imidazole (390 mg, $5.7 \mathrm{mmol})$, and TBDPSCl $(0.99 \mathrm{~mL}, 3.8 \mathrm{mmol})$ were added at $0{ }^{\circ} \mathrm{C}$ and stirred for $12 \mathrm{~h}$. After addition of $\mathrm{H}_{2} \mathrm{O}$, reaction mixture was diluted with EtOAc and washed with $\mathrm{H}_{2} \mathrm{O}$ three times. Organic layers were dried over $\mathrm{MgSO}_{4}$, filtered, evaporated, and purified by column chromatography on silica gel (EtOAc: $n$-hexane $=1: 10$ to 1:5) to afford TBDPS ether $8(1.4 \mathrm{~g}, 77 \%)$ as a colorless oil. IR $(\mathrm{KBr}) v_{\max }$ $3431,2934,2857,1692,1469,1424 \mathrm{~cm}^{-1},{ }^{1} \mathrm{H}-\mathrm{NMR}\left(\mathrm{CDCl}_{3}, 300 \mathrm{MHz}\right.$, mixture of rotamers) $\delta 7.67-7.65$ $(\mathrm{m}, 4 \mathrm{H}), 7.42-7.32(\mathrm{~m}, 6 \mathrm{H}), 5.66(\mathrm{dd}, 1 \mathrm{H}, J=4.8,15.6 \mathrm{~Hz}), 5.58-5.54(\mathrm{~m}, 1 \mathrm{H}), 4.76(\mathrm{~s}, 1 \mathrm{H}), 4.20(\mathrm{dd}, 2 \mathrm{H}$, $J=1.5,4.4 \mathrm{~Hz}), 3.90(\mathrm{~d}, 1 \mathrm{H}, J=12 \mathrm{~Hz}), 2.76(\mathrm{t}, 1 \mathrm{H}, J=12.7 \mathrm{~Hz}), 1.67-1.40(\mathrm{~m}, 6 \mathrm{H}), 1.42(\mathrm{~s}, 9 \mathrm{H}), 1.03(\mathrm{~s}$, 9H); ${ }^{13} \mathrm{C}$-NMR $\left(\mathrm{CDCl}_{3}, 100 \mathrm{MHz}\right.$, mixture of rotamers) $\delta 170.9,15.2,135.4,133.6,130.0,129.5,128.6$, $127.5,79.0,64.0,60.2,29.3,28.3,26.7,25.4,20.8,19.4,19.1,14.0$; LRMS (FAB) $m / z 480\left(\mathrm{M}+\mathrm{H}^{+}\right)$.

\subsection{7. (E)-1-(2-(3-(tert-butyldiphenylsilyloxy)prop-1-enyl)piperidin-1-yl)butan-1-one (9)}

To a solution of TBDPS ether 8 (62 mg, $0.13 \mathrm{mmol})$ in $\mathrm{CH}_{2} \mathrm{Cl}_{2}(2 \mathrm{~mL})$, trifluoroacetic acid (2 mL) was added and stirred for $30 \mathrm{~min}$. After evaporation of reaction mixture, the residue was dissolved in $\mathrm{CH}_{2} \mathrm{Cl}_{2}(2 \mathrm{~mL})$ and treated with $i \mathrm{Pr}_{2} \mathrm{NEt}(0.1 \mathrm{~mL})$ and n-butyryl chloride $(0.05 \mathrm{~mL})$ at $0{ }^{\circ} \mathrm{C}$ and stirred for $12 \mathrm{~h}$. Reaction mixture was quenched with $\mathrm{H}_{2} \mathrm{O}$ and extracted with $\mathrm{CH}_{2} \mathrm{Cl}_{2}$ twice. Organic layers were dried over $\mathrm{MgSO}_{4}$, filtered, evaporated, and purified by column chromatography on silica gel (EtOAc:n-hexane $=1: 3$ to 1:2) to afford butyryl amide $9(55 \mathrm{mg}, 95 \%)$ as a colorless oil. IR (KBr) $v_{\max }$ $2935,2858,1732,1644,1427,1238 \mathrm{~cm}^{-1} ;{ }^{1} \mathrm{H}-\mathrm{NMR}\left(\mathrm{CDCl}_{3}, 500 \mathrm{MHz}\right.$, mixture of rotamers) $\delta 7.68-7.62$ $(\mathrm{m}, 4 \mathrm{H}), 7.43-7.36(\mathrm{~m}, 6 \mathrm{H}), 5.68(\mathrm{~b}, 1 \mathrm{H}), 5.55(\mathrm{~d}, 1 \mathrm{H}, J=15.5 \mathrm{~Hz}), 4.52(\mathrm{~b}, 1 \mathrm{H}), 4.23(\mathrm{~s}, 1 \mathrm{H}), 3.70-3.59(\mathrm{~m}$, $1 \mathrm{H}), 3.07,2.60(\mathrm{~m}, 1 \mathrm{H}), 2.31(\mathrm{t}, 2 \mathrm{H}, J=7.5 \mathrm{~Hz}), 2.33-2.24(\mathrm{~m}, 1 \mathrm{H}), 1.75(\mathrm{~m}, 1 \mathrm{H}), 1.69-1.35(\mathrm{~m}, 7 \mathrm{H}), 1.06$ $(\mathrm{s}, 9 \mathrm{H}), 0.96(\mathrm{t}, 3 \mathrm{H}, J=7.5 \mathrm{~Hz}) ;{ }^{13} \mathrm{C}-\mathrm{NMR}\left(\mathrm{CDCl}_{3}, 125 \mathrm{M} \mathrm{Hz}\right) \delta 177.3,135.5,135.4,133.5,130.7,129.6$, 128.2, 127.6, 64.0, 63.7, 53.7, 48.7, 37.2, 35.8, 35.6, 35.1, 30.4, 28.9, 26.7, 26.4, 25.3, 19.5, 19.1, 18.9, 18.2, 13.9, 13.6; LRMS (FAB) $m / z 450\left(\mathrm{M}+\mathrm{H}^{+}\right)$; HRMS (FAB) calcd for $\mathrm{C}_{28} \mathrm{H}_{40} \mathrm{NO}_{7} \mathrm{Si}\left(\mathrm{M}+\mathrm{H}^{+}\right): 450.2828$, found 450.2832 .

\subsection{8. (E)-1-(2-(3-hydroxyprop-1-enyl)piperidin-1-yl)butan-1-one (10)}

To a solution of n-butyrl amide $9(370 \mathrm{mg}, 0.82 \mathrm{mmol})$ in THF $(10 \mathrm{~mL})$, acetic acid $(0.1 \mathrm{~mL}, 1.6 \mathrm{mmol})$ and TBAF (1.0 M in THF, $1.2 \mathrm{~mL}, 1.2 \mathrm{mmol}$ ) were added and stirred for $30 \mathrm{~min}$. After addition of aq. $\mathrm{NaHCO}_{3}$, reaction mixture was extracted with EtOAc three times. Organic layers were dried over $\mathrm{MgSO}_{4}$, filtered, evaporated and purified by column chromatography on silica gel (EtOAc:MeOH = 20:1) to afford primary alcohol $10(160 \mathrm{mg}, 92 \%)$ as a colorless oil. IR (KBr) $v_{\max } 3398,2936,2865,1620,1436,1253 \mathrm{~cm}^{-1}$; ${ }^{1} \mathrm{H}-\mathrm{NMR}\left(\mathrm{CDCl}_{3}, 400 \mathrm{MHz}\right.$, mixture of rotamers) $\delta 5.66-5.58(\mathrm{~m}, 2 \mathrm{H}), 5.31,4.49(\mathrm{bs}, 1 \mathrm{H}), 4.43,3.39(\mathrm{~d}$, $1 \mathrm{H}, J=12.8 \mathrm{~Hz}), 3.09,2.60(\mathrm{t}, 1 \mathrm{H}, J=12.4 \mathrm{~Hz}), 2.26(\mathrm{t}, 2 \mathrm{H}, J=7.2 \mathrm{~Hz}), 2.26-2.19(\mathrm{~m}, 1 \mathrm{H}), 1.74(\mathrm{~m}, 1 \mathrm{H})$, 1.64-1.54 (m, 7H), $1.34(\mathrm{~m}, 1 \mathrm{H}), 0.90(\mathrm{t}, 3 \mathrm{H}, J=6.0 \mathrm{~Hz}) ;{ }^{13} \mathrm{C}-\mathrm{NMR}\left(\mathrm{CDCl}_{3}, 100 \mathrm{MHz}\right.$, mixture of rotamers $)$ ठ 175.6, 172.4, 171.9, 131.2, 130.9, 129.3, 128.8, 62.9, 62.5, 53.6, 48.8, 41.8, 37.2, 35.5, 35.1, 30.2, 28.7, 26.1, 25.2, 19.4, 18.8, 13.9; LRMS (FAB) $m / z 212\left(\mathrm{M}+\mathrm{H}^{+}\right)$; HRMS (FAB) calcd for $\mathrm{C}_{12} \mathrm{H}_{22} \mathrm{NO}_{2}\left(\mathrm{M}+\mathrm{H}^{+}\right): 212.1651$, found 212.1644.

\subsection{9. (E)-1-(2-(3-(tert-butyldimethylsilyloxy)prop-1-enyl)piperidin-1-yl)butan-1-one (11)}

To a solution of alcohol 10 (72 mg, $0.34 \mathrm{mmol})$ in $\mathrm{CH}_{2} \mathrm{Cl}_{2}(2 \mathrm{~mL}), i \mathrm{Pr}_{2} \mathrm{NEt}(0.2 \mathrm{~mL})$ and TBSOTf $(0.05 \mathrm{~mL})$ were added at $0^{\circ} \mathrm{C}$ and stirred for $12 \mathrm{~h}$. After addition of $\mathrm{H}_{2} \mathrm{O}$, reaction mixture was extracted with $\mathrm{CH}_{2} \mathrm{Cl}_{2}$. Organic layers were dried over $\mathrm{MgSO}_{4}$, filtered, evaporated, and purified by column chromatography on silica gel (EtOAc: $n$-hexane $=1: 3$ to 1:2) to afford TBS ether $\mathbf{1 1}(110 \mathrm{mg}, 99 \%)$ as a colorless oil. IR (KBr) $v_{\max } 2934,2857,1645,1539,1424 \mathrm{~cm}^{-1} ;{ }^{1} \mathrm{H}-\mathrm{NMR}\left(\mathrm{CDCl}_{3}, 400 \mathrm{MHz}\right.$, mixture of rotamers) $\delta 5.59-5.49(\mathrm{~m}, 2 \mathrm{H}), 5.35(\mathrm{bs}), 3.59-3.57(\mathrm{~m}, 1 \mathrm{H}), 4.49-4.45(\mathrm{~m}, 1 \mathrm{H}), 4.13(\mathrm{~s}, 2 \mathrm{H}), 3.09,259$ (bs, 1H), $2.26(\mathrm{t}, 1 \mathrm{H}, J=5.6 \mathrm{~Hz}), 2.20(\mathrm{bs}, 1 \mathrm{H}), 1.73(\mathrm{~m}, 1 \mathrm{H}), 1.64-1.50(\mathrm{~m}, 6 \mathrm{H}), 1.34(\mathrm{~b}, 1 \mathrm{H}), 0.90(\mathrm{t}$, $3 \mathrm{H}, J=4.6 \mathrm{~Hz}), 0.85(\mathrm{~s}, 9 \mathrm{H}), 0.00(\mathrm{~s}, 6 \mathrm{H}) ;{ }^{13} \mathrm{C}-\mathrm{NMR}\left(\mathrm{CDCl}_{3}, 100 \mathrm{MHz}\right.$, mixture of rotamers) $\delta 172.2$, 
171.5, 131.1, 128.0, 127.8, 63.3, 62.9, 53.6, 48.6, 41.7, 37.2, 35.5, 35.1, 30.3, 28.8, 26.3, 25.8, 25.3, 19.5, 18.8, 18.2, 13.9; LRMS (FAB) $m / z 326\left(\mathrm{M}+\mathrm{H}^{+}\right)$; HRMS (FAB) calcd for $\mathrm{C}_{18} \mathrm{H}_{36} \mathrm{NO}_{2} \mathrm{Si}\left(\mathrm{M}+\mathrm{H}^{+}\right)$: 326.2515, found 326.2520 .

\subsubsection{0. (E)-1-(2-(3-(triethylsilyloxy)prop-1-enyl)piperidin-1-yl)butan-1-one (12)}

To a solution of alcohol 10 (100 mg, $0.47 \mathrm{mmol})$ in $\mathrm{CH}_{2} \mathrm{Cl}_{2}(2 \mathrm{~mL}), i \mathrm{Pr}_{2} \mathrm{NEt}(0.17 \mathrm{~mL}, 0.95 \mathrm{mmol})$ and TESCl (1.0 M in THF, $0.62 \mathrm{~mL}, 0.62 \mathrm{mmol}$ were added at $0{ }^{\circ} \mathrm{C}$ and stirred for $2 \mathrm{~h}$. After addition of $\mathrm{H}_{2} \mathrm{O}$, reaction mixture was extracted with $\mathrm{CH}_{2} \mathrm{Cl}_{2}$. Organic layers were dried over $\mathrm{MgSO}_{4}$, filtered, evaporated and purified by column chromatography on silica gel (EtOAc: $n$-hexane $=1: 3$ to 1:2) to afford TES ether $12(120 \mathrm{mg}, 78 \%)$ as a colorless oil. IR (KBr) $v_{\max } 2955,1645,1537,1458,1118 \mathrm{~cm}^{-1}$; ${ }^{1} \mathrm{H}-\mathrm{NMR}\left(\mathrm{CDCl}_{3}, 300 \mathrm{MHz}\right.$, mixture of rotamers) $85.57-5.36(\mathrm{~m}, 2 \mathrm{H}), 4.50(\mathrm{~b}, 1 \mathrm{H}), 4.13(\mathrm{~s}, 2 \mathrm{H}), 3.57-3.47$ $(\mathrm{m}), 3.41-3.37(\mathrm{~m}, 1 \mathrm{H}), 3.10(\mathrm{t}, J=12.0 \mathrm{~Hz}), 2.60(\mathrm{t}, 1 \mathrm{H}, J=12.0 \mathrm{~Hz}), 2.27(\mathrm{t}, 2 \mathrm{H}, J=7.5 \mathrm{~Hz}), 1.74-1.35(\mathrm{~m}$, $7 \mathrm{H}), 0.92(\mathrm{t}, 9 \mathrm{H}, J=8.1 \mathrm{~Hz}), 0.57(\mathrm{q}, 6 \mathrm{H}, J=8.1 \mathrm{~Hz})$; LRMS (FAB) $\mathrm{m} / z 326\left(\mathrm{M}+\mathrm{H}^{+}\right)$; HRMS (FAB) calcd for $\mathrm{C}_{18} \mathrm{H}_{36} \mathrm{NO}_{2} \mathrm{Si}\left(\mathrm{M}+\mathrm{H}^{+}\right)$: 326.2515, found 326.2519.

\subsubsection{1. (E)-1-(2-(3-(tert-butyldiphenylsilyloxy)prop-1-enyl)piperidin-1-yl)hex-5-yn-1-one (13)}

To a solution of TBDPS ether $8(72 \mathrm{mg}, 0.15 \mathrm{mmol})$ in $\mathrm{CH}_{2} \mathrm{Cl}_{2}(1 \mathrm{~mL})$, trifluoroacetic acid $(1 \mathrm{~mL})$ was added and stirred for $30 \mathrm{~min}$. After evaporation of reaction mixture, the residue was dissolved in $\mathrm{CH}_{2} \mathrm{Cl}_{2}(2 \mathrm{~mL})$ and treated with EDCI ( $\left.57 \mathrm{mg}, 0.3 \mathrm{mmol}\right), \mathrm{DMAP}(37 \mathrm{mg}, 0.3 \mathrm{mmol}$ ) and 5-hexynoic acid $(33 \mu \mathrm{L}, 0.3 \mathrm{mmol})$ at $0{ }^{\circ} \mathrm{C}$ and stirred for $12 \mathrm{~h}$. Reaction mixture was quenched with $\mathrm{H}_{2} \mathrm{O}$ and extracted with $\mathrm{CH}_{2} \mathrm{Cl}_{2}$ twice. Organic layers were dried over $\mathrm{MgSO}_{4}$, filtered, evaporated, and purified by column chromatography on silica gel (EtOAc: $n$-hexane $=1: 3$ to 1:2) to afford 5-hexynyl amide 13 (62 $\mathrm{mg}, 87 \%$ ) as a colorless oil. IR ( $\mathrm{KBr}) v_{\max } 3300,2935,2857,1642,1428,1256 \mathrm{~cm}^{-1},{ }^{1} \mathrm{H}-\mathrm{NMR}\left(\mathrm{CDCl}_{3}\right.$, $400 \mathrm{MHz}$, mixture of rotamers) $\delta 7.66-7.60(\mathrm{~m}, 4 \mathrm{H}), 7.42-7.24(\mathrm{~m}, 6 \mathrm{H}), 5.71-5.63(\mathrm{~m}, 1 \mathrm{H}), 5.57-5.50(\mathrm{~m}$, $1 \mathrm{H}), 4.53-4.45(\mathrm{~m}, 1 \mathrm{H}), 4.11(\mathrm{~d}, 2 \mathrm{H}, J=7.2 \mathrm{~Hz}), 3.69-3.60(\mathrm{~m}, 1 \mathrm{H}), 3.08-3.02(\mathrm{~m}, 1 \mathrm{H}), 2.47-2.44(\mathrm{~m}, 2 \mathrm{H})$, $2.32(\mathrm{~b}, 2 \mathrm{H}), 1.94-1.51(\mathrm{~m}, 9 \mathrm{H}), 1.12(\mathrm{~s}, 9 \mathrm{H}) ;{ }^{13} \mathrm{C}-\mathrm{NMR}\left(\mathrm{CDCl}_{3}, 100 \mathrm{MHz}\right.$, mixture of rotamers) $\delta 135.6$, 135.4, 133.5, 130.8, 129.6, 127.6, 83.8, 68.8, 63.8, 31.9, 31.4, 30.4, 28.9, 26.7, 26.3, 23.9, 19.5, 19.2 18.0; LRMS (FAB) $m / z 474\left(\mathrm{M}+\mathrm{H}^{+}\right)$; HRMS (FAB) calcd for $\mathrm{C}_{30} \mathrm{H}_{40} \mathrm{NO}_{7} \mathrm{Si}\left(\mathrm{M}+\mathrm{H}^{+}\right)$: 474.2828, found 474.2816.

\subsubsection{2. (E)-1-(2-(3-(tert-butyldiphenylsilyloxy)prop-1-enyl)piperidin-1-yl)hex-5-yn-1-one (14)}

To a solution of TBDPS ether $8(1.0 \mathrm{~g}, 2.1 \mathrm{mmol})$ in $\mathrm{CH}_{2} \mathrm{Cl}_{2}(10 \mathrm{~mL})$, trifluoroacetic acid $(5 \mathrm{~mL})$ was added and stirred for $1 \mathrm{~h}$. After addition of aq. $\mathrm{Na}_{2} \mathrm{CO}_{3}$, reaction mixture was extracted with $\mathrm{CH}_{2} \mathrm{Cl}_{2}$. Organic layers were dried over $\mathrm{MgSO}_{4}$, filtered, evaporated, and dissolved in $\mathrm{CH}_{2} \mathrm{Cl}_{2}(20 \mathrm{~mL})$. To this solution, 3-phenylpropionic acid $(0.5 \mathrm{~g}, 3.6 \mathrm{mmol})$, EDCI $(0.7 \mathrm{~g}, 3.6 \mathrm{mmol})$ and DMAP $(0.9 \mathrm{~g}$, $7.2 \mathrm{mmol}$ ) were added and stirred for $12 \mathrm{~h}$. Reaction mixture was quenched with aq. $\mathrm{NH}_{4} \mathrm{Cl}$ and extracted with $\mathrm{CH}_{2} \mathrm{Cl}_{2}$. Organic layers were dried over $\mathrm{MgSO}_{4}$, filtered, evaporated, and purified by column chromatography on silica gel (EtOAc: $n$-hexane $=1: 5)$ to afford amide $\mathbf{1 4}(0.9 \mathrm{~g}, 83 \%$ for 2 steps) as a pale yellow oil. ${ }^{1} \mathrm{H}-\mathrm{NMR}\left(\mathrm{CDCl}_{3}, 300 \mathrm{MHz}\right.$, mixture of rotamers) $\delta 7.77-7.63(\mathrm{~m}, 4 \mathrm{H}), 7.49-7.35(\mathrm{~m}$, $6 \mathrm{H}), 7.33-7.18(\mathrm{~m}, 5 \mathrm{H}), 5.74-5.43(\mathrm{~m}, 2 \mathrm{H}), 4.50(\mathrm{~s}, 1 \mathrm{H}), 4.25(\mathrm{~s}, 2 \mathrm{H}), 3.46(\mathrm{~m}, 1 \mathrm{H}), 3.00(\mathrm{t}, 2 \mathrm{H}, J=7.8$, $15.9 \mathrm{~Hz}), 2.67(\mathrm{t}, 2 \mathrm{H}, J=7.5,15.6 \mathrm{~Hz}), 1.48(\mathrm{~m}, 6 \mathrm{H}), 1.07(\mathrm{~m}, 10 \mathrm{H}) ;{ }^{13} \mathrm{C}-\mathrm{NMR}\left(\mathrm{CDCl}_{3}, 125 \mathrm{MHz}\right.$, mixture of rotamers) $\delta 71.6,170.9,141.3,135.5,134.7,134.6,133.6,130.9,129.7(2), 129.6,128.5,128.4,128.0,127.8$, 127.6, 127.4(2) 126.1, 77.3, 77.2, 77.0, 76.8, 64.1, 63.7, 53.9, 49.1, 41.8, 37.6, 34.9, 31.7, 30.3, 26.8, 26.2, 25.9, 25.8, 25.2, 19.5, 19.2, 18.6, 18.5. LRMS (EI) $m / z 512\left(\mathrm{M}+\mathrm{H}^{+}\right)$; HRMS (EI) calcd for $\mathrm{C}_{33} \mathrm{H}_{41} \mathrm{O}_{2} \mathrm{NSi}\left(\mathrm{M}^{+}\right)$: 511.2907, found 511.2903.

\subsubsection{3. (E)-tert-butyl 2-(3-(benzyloxy)prop-1-enyl)piperidine-1-carboxylate (15)}

To a solution of alcohol 7 (690 mg, $3.2 \mathrm{mmol})$ in THF (15 mL), TBAI (120 mg, $0.32 \mathrm{mmol}), \mathrm{NaH}(60 \%$ mineral oil, $150 \mathrm{mg}, 3.7 \mathrm{mmol})$ and benzyl bromide $(0.42 \mathrm{~mL}, 3.5 \mathrm{mmol})$ were added at $0{ }^{\circ} \mathrm{C}$ and stirred for $12 \mathrm{~h}$ at room temperature. After addition of $\mathrm{H}_{2} \mathrm{O}$, reaction mixture was extracted with EtOAc twice. Organic layers were dried over $\mathrm{MgSO}_{4}$, filtered, evaporated and purified by column chromatography 
on silica gel (EtOAc: $n$-hexane $=1: 10$ to 1:5) to afford benzyl ether $15(920 \mathrm{mg}, 86 \%)$ as a colorless oil. IR (KBr) $v_{\max } 2935,2857,1691,1453,1409,1365 \mathrm{~cm}^{-1} ;{ }^{1} \mathrm{H}-\mathrm{NMR}\left(\mathrm{CDCl}_{3}, 400 \mathrm{MHz}\right.$, mixture of rotamers) $\delta$ 7.31-7.22 (m, 5H), 5.68-5.55 (m, 2H), $4.80(\mathrm{~s}, 1 \mathrm{H}), 4.48(\mathrm{~d}, 2 \mathrm{H}, J=4.8 \mathrm{~Hz}), 4.01-4.00(\mathrm{~m}, 2 \mathrm{H}), 3.92(\mathrm{~d}, 1 \mathrm{H}$, $J=13.2 \mathrm{~Hz}), 2.80(\mathrm{t}, 1 \mathrm{H}, J=11.6 \mathrm{~Hz}), 1.72-1.62(\mathrm{~m}, 2 \mathrm{H}), 1.60-1.50(\mathrm{~m}, 2 \mathrm{H}), 1.47-1.31(\mathrm{~m}, 2 \mathrm{H}), 1.43(\mathrm{~s}$, 9H); ${ }^{13} \mathrm{C}-\mathrm{NMR}\left(\mathrm{CDCl}_{3}, 100 \mathrm{MHz}\right.$, mixture of rotamers) $\delta 155.0,138.1,131.8,128.2,127.5,127.4,79.1$, 72.3, 71.7, 70.2, 66.1, 39.6, 28.9, 28.3, 25.3, 19.4; LRMS (FAB) $\mathrm{m} / \mathrm{z} 332\left(\mathrm{M}+\mathrm{H}^{+}\right)$; HRMS (FAB) calcd for $\mathrm{C}_{20} \mathrm{H}_{30} \mathrm{NO}_{3}\left(\mathrm{M}+\mathrm{H}^{+}\right)$: 332.2226, found 332.2226.

\subsubsection{4. (E)-1-(2-(3-(benzyloxy)prop-1-enyl)piperidin-1-yl)propan-1-one (16)}

Benzyl ether 15 (120 mg, $0.36 \mathrm{mmol}$ ) was converted to propyl amide 16 (75 mg, 72\%) using same procedure as described in Section 3.1.7. IR (KBr) $v_{\max } 2936,2857,1644,1538,1426,1251 \mathrm{~cm}^{-1}$; ${ }^{1} \mathrm{H}-\mathrm{NMR}$ $\left(\mathrm{CDCl}_{3}, 400 \mathrm{MHz}\right.$, mixture of rotamers) $\delta 7.33-7.11(\mathrm{~m}, 5 \mathrm{H}), 5.70-5.55(\mathrm{~m}, 2 \mathrm{H}), 5.39(\mathrm{bs}), 3.60(\mathrm{~d}, 1 \mathrm{H}, J=$ $11.8 \mathrm{~Hz}), 4.48(\mathrm{~s}, 2 \mathrm{H}), 4.48(\mathrm{bs}, 1 \mathrm{H}), 3.97(\mathrm{~d}, 2 \mathrm{H}, J=5.8 \mathrm{~Hz}), 3.09(\mathrm{t}, J=12.3 \mathrm{~Hz}), 2.62(\mathrm{t}, 1 \mathrm{H}, J=12.3 \mathrm{~Hz})$, 2.34-2.27 (m, 2H), 1.79-1.76 (m, 1H), 1.63-1.52 (m, 4H), $1.27(\mathrm{~m}, 1 \mathrm{H}), 1.09(\mathrm{t}, 3 \mathrm{H}, J=6.0 \mathrm{~Hz}) ;{ }^{13} \mathrm{C}-\mathrm{NMR}$ $\left(\mathrm{CDCl}_{3}, 100 \mathrm{MHz}\right.$, mixture of rotamers) $\delta 173.0,172.4,138.1,131.4,130.9,128.3,128.0,127.6,126.9,72.2$, 72.0, 70.3, 69.9, 53.5, 48.8, 41.6, 37.3, 30.1, 28.5, 26.7, 26.2, 25.2, 19.6, 9.4, 9.0; LRMS (FAB) $\mathrm{m} / \mathrm{z} 288$ (M + $\mathrm{H}^{+}$); HRMS (FAB) calcd for $\mathrm{C}_{18} \mathrm{H}_{26} \mathrm{NO}_{2}\left(\mathrm{M}+\mathrm{H}^{+}\right)$: 288.1964, found 288.1956.

\subsubsection{5. (E)-1-(2-(3-(benzyloxy)prop-1-enyl)piperidin-1-yl)butan-1-one (17)}

Benzyl ether 15 (140 mg, $0.42 \mathrm{mmol}$ ) was converted to butyl amide 17 (97 mg, 77\%) using same procedure as described in Section 3.1.7. IR (KBr) $v_{\max } 2935,2858,1641,1425,1362 \mathrm{~cm}^{-1}, 1 \mathrm{H}-\mathrm{NMR}$ $\left(\mathrm{CDCl}_{3}, 400 \mathrm{MHz}\right.$, mixture of rotamers) $87.33-7.23(\mathrm{~m}, 5 \mathrm{H}), 5.70-5.60(\mathrm{~m}, 2 \mathrm{H}), 5.39(\mathrm{bs}), 3.60(\mathrm{~d}, 1 \mathrm{H}, J=$ $9.0 \mathrm{~Hz}), 4.48(\mathrm{~m}, 3 \mathrm{H}), 4.01(\mathrm{~d}, 2 \mathrm{H}, J=3.2 \mathrm{~Hz}), 3.09(\mathrm{t}, J=12.3 \mathrm{~Hz}), 2.58(\mathrm{t}, 1 \mathrm{H}, J=12.3 \mathrm{~Hz}), 2.31-2.21$ $(\mathrm{m}, 2 \mathrm{H}), 1.79-1.76(\mathrm{~m}, 1 \mathrm{H}), 1.67-1.58(\mathrm{~m}, 6 \mathrm{H}), 1.36(\mathrm{~m}, 1 \mathrm{H}), 0.93(\mathrm{t}, 3 \mathrm{H}, J=7.2 \mathrm{~Hz}) ;{ }^{13} \mathrm{C}-\mathrm{NMR}\left(\mathrm{CDCl}_{3}\right.$, $100 \mathrm{MHz}$, mixture of rotamers) $\delta 176.6,172.3,171.8,138.1,137.9,131.4,130.9,128.3,127.7,127.6,72.2$, 71.9, 70.3, 69.9, 53.6, 48.7, 41.8, 37.2, 35.8, 35.5, 35.1, 30.2, 28.6, 26.2, 25.2, 19.6, 19.4, 18.8, 18.6, 18.2, 13.9, 13.5; LRMS (FAB) $m / z 302\left(\mathrm{M}+\mathrm{H}^{+}\right)$; HRMS (FAB) calcd for $\mathrm{C}_{19} \mathrm{H}_{28} \mathrm{NO}_{2}\left(\mathrm{M}+\mathrm{H}^{+}\right)$: 302.2120, found 302.2120 .

\subsubsection{6. (E)-1-(2-(3-(benzyloxy)prop-1-enyl)piperidin-1-yl)pentan-1-one (18)}

Benzyl ether 15 (190 mg, $0.57 \mathrm{mmol}$ ) was converted to pentyl amide 18 (99 mg, 55\%) using same procedure as described in Section 3.1.7. IR (KBr) $v_{\max } 2933,2858,1642,1424,1263 \mathrm{~cm}^{-1}$; ${ }^{1} \mathrm{H}-\mathrm{NMR}$ $\left(\mathrm{CD}_{3} \mathrm{OD}, 400 \mathrm{MHz}\right.$, mixture of rotamers) $\delta 7.32-7.23(\mathrm{~m}, 5 \mathrm{H}), 5.80-5.56(\mathrm{~m}, 2 \mathrm{H}), 5.29(\mathrm{bs}), 4.67(\mathrm{bs}, 1 \mathrm{H})$, $4.49(\mathrm{~s}, 2 \mathrm{H}), 4.40(\mathrm{~d}, J=12.3 \mathrm{~Hz}), 3.73(\mathrm{~d}, 1 \mathrm{H}, J=12.4 \mathrm{~Hz}), 4.01(\mathrm{~d}, 2 \mathrm{H}, J=3.2 \mathrm{~Hz}), 3.15(\mathrm{t}, J=12.9 \mathrm{~Hz})$, $2.58(\mathrm{t}, 1 \mathrm{H}, J=12.5 \mathrm{~Hz}), 2.45-2.25(\mathrm{~m}, 2 \mathrm{H}), 1.82(\mathrm{~b}, 1 \mathrm{H}), 1.68-1.53(\mathrm{~m}, 6 \mathrm{H}), 1.39-1.20(\mathrm{~m}, 3 \mathrm{H}), 0.93-0.86$ $(\mathrm{m}, 3 \mathrm{H}) ;{ }^{13} \mathrm{C}-\mathrm{NMR}\left(\mathrm{CDCl}_{3}, 100 \mathrm{MHz}\right.$, mixture of rotamers) $\delta 176.4,172.4,1719,138.1,131.4,131.0,128.3$, 128.0, 127.5, 72.0, 70.3, 69.9, 53.7, 48.7, 41.8, 37.3, 33.6, 33.3, 32.9, 30.2, 28.6, 27.5, 26.8, 26.3, 25.2, 22.5, 22.1, 19.6, 14.0, 13.6; LRMS (FAB) $m / z 316\left(\mathrm{M}+\mathrm{H}^{+}\right)$; HRMS (FAB) calcd for $\mathrm{C}_{20} \mathrm{H}_{30} \mathrm{NO}_{2}\left(\mathrm{M}+\mathrm{H}^{+}\right): 316.2277$, found 316.2278 .

\subsubsection{N-(4-methoxybenzyl)butyramide (20)}

To a solution of 4-methoxybenzylamine 19 (300 mg, $2.2 \mathrm{mmol})$ in $\mathrm{CH}_{2} \mathrm{Cl}_{2}(10 \mathrm{~mL}), \mathrm{Et}_{3} \mathrm{~N}(0.4 \mathrm{~mL}$, $2.8 \mathrm{mmol})$ and n-butyryl chloride $(0.25 \mathrm{~mL}, 2.4 \mathrm{mmol})$ were at $0{ }^{\circ} \mathrm{C}$ and stirred for $5 \mathrm{~h}$ at room temperature. Reaction mixture was quenched with $\mathrm{H}_{2} \mathrm{O}$ and extracted with $\mathrm{CH}_{2} \mathrm{Cl}_{2}$ twice. Organic layers were dried over $\mathrm{MgSO}_{4}$, filtered, evaporated, and purified by column chromatography on silica gel (EtOAc: $n$-hexane $=1: 1)$ to afford para-nethoxyl benzyl (PMB) amide $20(320 \mathrm{mg}, 71 \%)$ as a white amorphous solid. IR (KBr) $v_{\max } 3290,2959,1632,1554,1513 \mathrm{~cm}^{-1} ;{ }^{1} \mathrm{H}-\mathrm{NMR}\left(\mathrm{CDCl}_{3}, 400 \mathrm{M} \mathrm{Hz}\right) \delta 7.17$ $(\mathrm{d}, 2 \mathrm{H}, J=8.6 \mathrm{~Hz}), 6.83(\mathrm{~d}, 2 \mathrm{H}, J=8.6 \mathrm{~Hz}), 4.85(\mathrm{~s}, 1 \mathrm{H}), 4.26(\mathrm{~s}, 2 \mathrm{H}), 3.72(\mathrm{~s}, 3 \mathrm{H}), 2.17(\mathrm{t}, 2 \mathrm{H}, J=7.2 \mathrm{~Hz})$, 1.67-1.58 (m, 2H), $0.92(\mathrm{t}, 3 \mathrm{H}, J=7.3 \mathrm{~Hz}) ;{ }^{13} \mathrm{C}-\mathrm{NMR}\left(\mathrm{CDCl}_{3}, 100 \mathrm{M} \mathrm{Hz}\right) \delta 176.4,161.0,132.8,130.6,115.6$, 
56.4, 44.4, 39.7, 21.1, 14.8; LRMS (FAB) $m / z 208\left(\mathrm{M}+\mathrm{H}^{+}\right)$; HRMS (FAB) calcd for $\mathrm{C}_{12} \mathrm{H}_{18} \mathrm{NO}_{2}\left(\mathrm{M}+\mathrm{H}^{+}\right)$: 208.1338, found 208.1334.

\subsubsection{8. $\mathrm{N}$-(but-2-enyl)-N-(4-methoxybenzyl)butyramide (21)}

To a solution of PMB amide 20 (115 mg, $0.55 \mathrm{mmol})$ in DMF (3 mL), NaH (60\% mineral oil, $26 \mathrm{mg}$, $0.66 \mathrm{mmol})$ and trans-crotyl bromide $(0.10 \mathrm{~mL}, 0.83 \mathrm{mmol})$ were added at $0{ }^{\circ} \mathrm{C}$ and stirred for $2 \mathrm{~h}$ at room temperature. After dilution with EtOAc and reaction mixture was washed with $\mathrm{H}_{2} \mathrm{O} 3$ times. Organic layers were dried over $\mathrm{MgSO}_{4}$, filtered, evaporated, and purified by column chromatography on silica gel (EtOAc: $n$-hexane $=1: 3)$ to afford crotyl amide $21(128 \mathrm{mg}, 87 \%)$ as a colorless oil. IR $(\mathrm{KBr})$ $v_{\max } 2962,1645,1513,1459,1248 \mathrm{~cm}^{-1} ;{ }^{1} \mathrm{H}-\mathrm{NMR}\left(\mathrm{CDCl}_{3}, 400 \mathrm{MHz}\right.$, mixtures of rotamers) $\delta 7.14-7.12$ $(\mathrm{m}, 2 \mathrm{H}), 6.85-6.78(\mathrm{~m}, 2 \mathrm{H}), 5.60-5.45(\mathrm{~m}, 1 \mathrm{H}), 5.44-5.27(\mathrm{~m}, 1 \mathrm{H}), 4.46(\mathrm{~s}), 4.39(\mathrm{~s}, 2 \mathrm{H}), 3.99(\mathrm{~d}, J=6.8 \mathrm{~Hz})$, $3.79(\mathrm{~d}, 1 \mathrm{H}, J=6.8 \mathrm{~Hz}), 3.87(\mathrm{~d}, J=6.0 \mathrm{~Hz}), 3.68(\mathrm{~d}, 1 \mathrm{H}, J=6.0 \mathrm{~Hz}), 3.76(\mathrm{~s}), 3.74(\mathrm{~s}, 3 \mathrm{H}), 2.32-2.27(\mathrm{~m}$, 2H), 1.71-1.50 (m, 5H), 0.96-0.84 (m, 3H); ${ }^{13} \mathrm{C}-\mathrm{NMR}\left(\mathrm{CDCl}_{3}, 100 \mathrm{MHz}\right.$, mixtures of rotamers) $\delta 172.9$, $172.8,158.8,158.7,130.0,129.8,129.4,128.8,128.7,128.1,127.7,127.5,127.4,127.3,125.9,125.8,125.5$, 114.1, 113.7, 55.1, 49.4, 49.1, 48.1, 47.3, 47.0, 46.7, 43.4, 41.2, 35.1, 34.9, 18.7, 17.6, 17.5, 13.9, 12.8; LRMS (FAB) $m / z 262\left(\mathrm{M}+\mathrm{H}^{+}\right)$; HRMS (FAB) calcd for $\mathrm{C}_{16} \mathrm{H}_{24} \mathrm{NO}_{2}\left(\mathrm{M}+\mathrm{H}^{+}\right)$: 262.1807, found 262.1800.

3.1.19. (Rac-3S,4R,E)-4-((tert-butyldiphenylsilyloxy)methyl)-3-ethyl-3,4,7,8,9,10-hexahydroazecin2(1H)-one $\left(9^{\prime}\right)$

TBDPS ether 9 (27 $\mathrm{mg}, 0.060 \mathrm{mmol}$ ) was converted to macrolactam $\mathbf{9}^{\prime}$ as an amorphous solid using Procedure A (13 mg, 48\%) or Procedure B (2 mg, 7.5\%). IR (KBr) $v_{\max } 3300,2929,1645,1541,1458 \mathrm{~cm}^{-1}$; ${ }^{1} \mathrm{H}-\mathrm{NMR}\left(\mathrm{CDCl}_{3}, 300 \mathrm{M} \mathrm{Hz}\right)$ 8 7.58-7.56 (m, 4H), 7.36-7.29 (m, 6H), 5.49-5.36 (m, 2H), $4.89(\mathrm{~d}, 1 \mathrm{H}, J=$ $9.0 \mathrm{~Hz}), 3.71-3.56(\mathrm{~m}, 3 \mathrm{H}), 2.77(\mathrm{dd}, 1 \mathrm{H}, J=6.9,12.9 \mathrm{~Hz}), 2.13(\mathrm{~m}, 2 \mathrm{H}), 1.97(\mathrm{dt}, 2 \mathrm{H}, J=7.2,11.6 \mathrm{~Hz})$, $1.89-1.13(\mathrm{~m}, 7 \mathrm{H}), 1.00(\mathrm{~s}, 3 \mathrm{H}), 0.76(\mathrm{t}, 3 \mathrm{H}, J=7.2 \mathrm{~Hz}) ;{ }^{13} \mathrm{C}-\mathrm{NMR}\left(\mathrm{CDCl}_{3}, 75 \mathrm{M} \mathrm{Hz}\right) \delta 174.8,135.6,133.6$, 133.2, 130.4, 129.7, 127.6, 63.7, 53.5, 49.0, 40.3, 33.1, 29.8, 26.9, 26.8, 21.4, 19.4, 12.8; LRMS (FAB) $m / z 450$ $\left(\mathrm{M}+\mathrm{H}^{+}\right)$; HRMS (FAB) calcd for $\mathrm{C}_{28} \mathrm{H}_{40} \mathrm{NO}_{7} \mathrm{Si}\left(\mathrm{M}+\mathrm{H}^{+}\right)$: 450.2828, found 450.2832.

3.1.20. (Rac-3S,4R,E)-4-((tert-buyldimethylsilyloxy)methyl)-3-ethyl-3,4,7,8,9,10hexahydroazecin-2(1H)-one (11')

TBS ether 11 (48 $\mathrm{mg}, 0.15 \mathrm{mmol}$ ) was converted to macrolactam 11' as an amorphous solid using Procedure A (33 mg, 69\%) or Procedure B (12 mg, 25\%). IR (KBr) $v_{\max } 3294,2928,2857,1644,1550 \mathrm{~cm}^{-1}$; ${ }^{1} \mathrm{H}-\mathrm{NMR}\left(\mathrm{CDCl}_{3}, 400 \mathrm{M} \mathrm{Hz}\right) \delta 5.43-5.36(\mathrm{~m}, 2 \mathrm{H}), 4.97(\mathrm{~d}, 1 \mathrm{H}, J=5.2 \mathrm{~Hz}), 3.71-3.62(\mathrm{~m}, 3 \mathrm{H}), 2.80(\mathrm{dd}$, $1 \mathrm{H}, J=6.0,10.8 \mathrm{~Hz}), 2.21-2.13(\mathrm{~m}, 2 \mathrm{H}), 1.97(\mathrm{dt}, 1 \mathrm{H}, J=3.1,6.5 \mathrm{~Hz}), 1.92-1.86(\mathrm{~m}, 1 \mathrm{H}), 1.81-1.76(\mathrm{~m}$, $1 \mathrm{H}), 1.71-1.62(\mathrm{~m}, 2 \mathrm{H}), 1.49-1.42(\mathrm{~m}, 2 \mathrm{H}), 1.24-1.17(\mathrm{~m}, 1 \mathrm{H}), 0.87(\mathrm{~s}, 9 \mathrm{H}), 0.84(\mathrm{t}, 3 \mathrm{H}, J=5.8 \mathrm{~Hz}), 0.01(\mathrm{~s}$, $3 \mathrm{H}), 0.01(\mathrm{~s}, 3 \mathrm{H}) ;{ }^{13} \mathrm{C}-\mathrm{NMR}\left(\mathrm{CD}_{3} \mathrm{OD}, 100 \mathrm{M} \mathrm{Hz}\right) \delta 178.4,134.3,133.3,65.4,54.8,51.6,42.0,34.4,31.4$, 27.2, 23.1, 20.0, 13.9, -4.3, -4.5; LRMS (FAB) $\mathrm{m} / z 326\left(\mathrm{M}+\mathrm{H}^{+}\right.$); HRMS (FAB) calcd for $\mathrm{C}_{18} \mathrm{H}_{36} \mathrm{NO}_{2} \mathrm{Si}$ $\left(\mathrm{M}+\mathrm{H}^{+}\right): 326.2515$, found 326.2514 .

\subsubsection{1. (Rac-3S,4R,E)-4-((triethylsilyloxy)methyl)-3-ethyl-3,4,7,8,9,10-hexahydroazecin-2(1H)-one (12')}

TES ether 12 (40 mg, $0.12 \mathrm{mmol}$ ) was converted to macrolactam 12' as an amorphous solid using Procedure A (31 mg, 69\%). IR (KBr) $v_{\max } 3299,2926,1646,1553,1453,137 \mathrm{~cm}^{-1} ;{ }^{1} \mathrm{H}-\mathrm{NMR}\left(\mathrm{CDCl}_{3}\right.$, $400 \mathrm{M} \mathrm{Hz}) \delta 5.45-5.35(\mathrm{~m}, 2 \mathrm{H}), 5.00(\mathrm{~d}, 1 \mathrm{H}, J=8.3 \mathrm{~Hz}), 3.71-3.60(\mathrm{~m}, 3 \mathrm{H}), 2.80(\mathrm{dd}, 1 \mathrm{H}, J=7.6,13.7 \mathrm{~Hz})$, $2.21-2.00(\mathrm{~m}, 2 \mathrm{H}), 1.96(\mathrm{dt}, 1 \mathrm{H}, J=4.0,10.2 \mathrm{~Hz}), 1.92-1.87(\mathrm{~m}, 1 \mathrm{H}), 1.81-1.67(\mathrm{~m}, 3 \mathrm{H}), 1.50-1.42(\mathrm{~m}, 2 \mathrm{H})$, $1.25-1.16(\mathrm{~m}, 1 \mathrm{H}), 0.92(\mathrm{t}, 9 \mathrm{H}, J=8.0 \mathrm{~Hz}), 0.84(\mathrm{t}, 3 \mathrm{H}, J=7.2 \mathrm{~Hz}), 0.56(\mathrm{q}, 6 \mathrm{H}, J=8.0 \mathrm{~Hz}) ;{ }^{13} \mathrm{C}-\mathrm{NMR}$ $\left(\mathrm{CDCl}_{3}, 100 \mathrm{M} \mathrm{Hz}\right) \delta$ 174.9, 133.0, 130.5, 77.2, 62.5, 53.1, 49.0, 40.3, 33.1, 29.7, 21.4, 12.8, 6.7, 4.3; LRMS (FAB) $m / z 326\left(\mathrm{M}+\mathrm{H}^{+}\right)$; HRMS (FAB) calcd for $\mathrm{C}_{18} \mathrm{H}_{36} \mathrm{NO}_{2} \mathrm{Si}\left(\mathrm{M}+\mathrm{H}^{+}\right)$: 326.2515, found 326.2519. 
3.1.22. (Rac-3S,4R,E)- 3-(but-3-ynyl)-4-((tert-butyldiphenylsilyloxy)methyl)-3,4,7,8,9,10hexahydroazecin-2(1H)-one (13')

Hexynyl amide $13(22 \mathrm{mg}, 46 \mu \mathrm{mol})$ was converted to macrolactam $13^{\prime}$ as an amorphous solid using Procedure A $(12 \mathrm{mg}, 55 \%)$. IR (KBr) $v_{\max } 3303,2928,2857,1645,1541,1430 \mathrm{~cm}^{-1},{ }^{1} \mathrm{H}-\mathrm{NMR}\left(\mathrm{CDCl}_{3}\right.$, $300 \mathrm{MHz}$ ) $\delta 7.67-7.63(\mathrm{~m}, 4 \mathrm{H}), 7.44-7.33(\mathrm{~m}, 6 \mathrm{H}), 5.51(\mathrm{dd}, 1 \mathrm{H}, J=8.7,15.6 \mathrm{~Hz}), 5.46-5.36(\mathrm{~m}, 1 \mathrm{H})$, $5.01(\mathrm{~d}, 1 \mathrm{H}, J=9.6 \mathrm{~Hz}), 3.71(\mathrm{~d}, 2 \mathrm{H}, J=2.7 \mathrm{~Hz}), 3.67(\mathrm{~m}, 1 \mathrm{H}), 2.81(\mathrm{dd}, 1 \mathrm{H}, J=7.5,13.2 \mathrm{~Hz}), 2.44(\mathrm{dd}$, $1 \mathrm{H}, J=3.0,10,8 \mathrm{~Hz}), 2.29-2.15(\mathrm{~m}, 3 \mathrm{H}), 2.10-1.47(\mathrm{~m}, 8 \mathrm{H}), 1.23(\mathrm{~m}, 1 \mathrm{H}), 1.05(\mathrm{~s}, 9 \mathrm{H}) ;{ }^{13} \mathrm{C}-\mathrm{NMR}\left(\mathrm{CDCl}_{3}\right.$, $75 \mathrm{M} \mathrm{Hz}) \delta 174.1,135.7,135.6,133.5,133.4,130.0,129.7,129.6,127.7,84.0,68.6,63.4,50.0,48.9,40.3,33.1$, 27.0, 26.9, 19.3, 17.0; LRMS (FAB) $m / z 474\left(\mathrm{M}+\mathrm{H}^{+}\right)$; HRMS (FAB) calcd for $\mathrm{C}_{30} \mathrm{H}_{40} \mathrm{NO}_{7} \mathrm{Si}\left(\mathrm{M}+\mathrm{H}^{+}\right)$: 474.2828 , found 474.2832 .

3.1.23. (Rac-3S, $4 R, E)-$

3-benzyl-4-((tert-butyldiphenylsilyloxy)methyl)-3,4,7,8,9,10-hexahydroazecin-2(1H)-one (14')

Phenylethyl amide $14(1.0 \mathrm{~g}, 1.9 \mathrm{mmol})$ was converted to macrolactam $14^{\prime}$ as an amorphous solid using Procedure A (0.94 g, 94\%). ${ }^{1} \mathrm{H}-\mathrm{NMR}\left(\mathrm{CDCl}_{3}, 500 \mathrm{M} \mathrm{Hz}\right) \delta 7.62-7.59(\mathrm{~m}, 4 \mathrm{H}), 7.37-7.25(\mathrm{~m}, 6 \mathrm{H})$, 7.14-7.02 (m, 5H), 5.46-5.36 (m, 2H), $4.82(\mathrm{~d}, 1 \mathrm{H}, J=8.0 \mathrm{~Hz}), 3.84-3.75(\mathrm{~m}, 2 \mathrm{H}), 3.51(\mathrm{ddd}, 1 \mathrm{H}, J=9.0$, 12.5, 17.0 Hz), $2.93(\mathrm{dd}, 1 \mathrm{H}, J=12.0,14.0 \mathrm{~Hz}), 2.61(\mathrm{dd}, 2 \mathrm{H}, J=2.5,13.5 \mathrm{~Hz}), 2.57(\mathrm{dd}, 1 \mathrm{H}, J=11.5$, $19 \mathrm{~Hz}), 2.39(\mathrm{ddd}, 1 \mathrm{H}, J=2.5,10.5,13 \mathrm{~Hz}), 2.24(\mathrm{dd}, 1 \mathrm{H}, J=8.5,18.5, \mathrm{~Hz}), 2.14(\mathrm{dd}, 1 \mathrm{H}, J=3.5,12.5, \mathrm{~Hz})$ $1.83-1.71(\mathrm{~m}, 2 \mathrm{H}), 1.63(\mathrm{~d}, 1 \mathrm{H}, J=7.0 \mathrm{~Hz}), 1.40(\mathrm{dd}, 1 \mathrm{H}, J=11.5,23.0 \mathrm{~Hz}), 1.06-0.99(\mathrm{~m}, 9 \mathrm{H}) ;{ }^{13} \mathrm{C}-\mathrm{NMR}$ $\left(\mathrm{CDCl}_{3}, 125 \mathrm{M} \mathrm{Hz}\right) 8173.9,141.0,140.7,135.7,133.5,129.9,128.9,128.5,127.7,127.0,125.9,65.2,63.6,53.8$, 49.0, 40.3, 34.3, 33.1, 29.8, 27.0, 19.4; LRMS (EI) $\mathrm{m} / \mathrm{z} 512\left(\mathrm{M}+\mathrm{H}^{+}\right)$; HR-MS (EI) calcd for $\mathrm{C}_{33} \mathrm{H}_{41} \mathrm{O}_{2} \mathrm{NSi}$ $\left(\mathrm{M}^{+}\right): 511.2907$, found 511.2908.

\subsubsection{4. (Rac-3S,4R,E)- 4-(benzyloxymethyl)-3-methyl-3,4,7,8,9,10-hexahydroazecin-2(1H)-one (16')}

Propyl amide 16 (20 mg, $70 \mu \mathrm{mol}$ ) was converted to macrolactam 16' as an amorphous solid using Procedure A (16 mg, 80\%) or Procedure B (7 mg, 35\%). IR (KBr) $v_{\max } 3312,2924,1644,1550$, 1452, $1367 \mathrm{~cm}^{-1} ;{ }^{1} \mathrm{H}-\mathrm{NMR}\left(\mathrm{CD}_{3} \mathrm{OD}, 300 \mathrm{M} \mathrm{Hz}\right) \delta 7.33-7.24(\mathrm{~m}, 5 \mathrm{H}), 5.52-5.31(\mathrm{~m}, 2 \mathrm{H}), 4.48(\mathrm{q}, 2 \mathrm{H}$, $J=12.0 \mathrm{~Hz}), 3.54(\mathrm{~d}, 2 \mathrm{H}, J=4.5 \mathrm{~Hz}), 3.57-3.47(\mathrm{~m}, 1 \mathrm{H}), 2.73(\mathrm{dd}, 1 \mathrm{H}, J=6.9,13.5 \mathrm{~Hz}), 2.37-2.12(\mathrm{~m}, 3 \mathrm{H})$, 1.93-1.72 (m, 3H), 1.51-1.27 (m, 2H), $1.05(\mathrm{~d}, 3 \mathrm{H}, J=6.3 \mathrm{~Hz}) ;{ }^{13} \mathrm{C}-\mathrm{NMR}\left(\mathrm{CDCl}_{3}, 100 \mathrm{M} \mathrm{Hz}\right) \delta 175.6$, 138.2, 133.8, 130.3, 128.3, 127.6, 127.5, 126.9, 73.2, 69.7, 48.5, 45.0, 40.1, 33.0, 29.6, 13.6; LRMS (FAB) $\mathrm{m} / \mathrm{z}$ $288\left(\mathrm{M}+\mathrm{H}^{+}\right)$; HRMS (FAB) calcd for $\mathrm{C}_{18} \mathrm{H}_{26} \mathrm{NO}_{2}\left(\mathrm{M}+\mathrm{H}^{+}\right)$: 288.1964, found 288.1956.

\subsubsection{5. (Rac-3S,4R,E)-4-(benzyloxymethyl)-3-ethyl-3,4,7,8,9,10-hexahydroazecin-2(1H)-one (17')}

Butyryl amide $17(20 \mathrm{mg}, 66 \mu \mathrm{mol})$ was converted to macrolactam $\mathbf{1 7}^{\prime}$ as an amorphous solid using Procedure A (14 mg, 70\%) or Procedure B (3 mg, 15\%). IR (KBr) $v_{\max } 2926,2856,2421,1637,1457 \mathrm{~cm}^{-1}$; ${ }^{1} \mathrm{H}-\mathrm{NMR}\left(\mathrm{CD}_{3} \mathrm{OD}, 300 \mathrm{M} \mathrm{Hz}\right) \delta 7.24-7.15(\mathrm{~m}, 5 \mathrm{H}), 5.41-5.22(\mathrm{~m}, 2 \mathrm{H}), 4.38(\mathrm{q}, 2 \mathrm{H}, J=12.0 \mathrm{~Hz}), 3.44$ $(\mathrm{d}, 2 \mathrm{H}, J=4.0 \mathrm{~Hz}), 3.45-3.44(\mathrm{~m}, 1 \mathrm{H}), 2.66(\mathrm{~m}, 1 \mathrm{H}), 2.16-2.02(\mathrm{~m}, 3 \mathrm{H}), 1.78-1.18(\mathrm{~m}, 7 \mathrm{H}), 0.73(\mathrm{t}, 3 \mathrm{H}$, $J=7.2 \mathrm{~Hz}) ;{ }^{13} \mathrm{C}-\mathrm{NMR}\left(\mathrm{CD}_{3} \mathrm{OD}, 75 \mathrm{M} \mathrm{Hz}\right) \delta 178.2,140.5,134.5,133.3,130.1,129.7,129.4,74.9,72.3,55.0$, 42.0, 33.9, 31.4, 23.1, 21.4, 14.9, 13.8.; LRMS (FAB) $\mathrm{m} / \mathrm{z} 302\left(\mathrm{M}+\mathrm{H}^{+}\right)$; HRMS (FAB) calcd for $\mathrm{C}_{19} \mathrm{H}_{28} \mathrm{NO}_{2}$ $\left(\mathrm{M}+\mathrm{H}^{+}\right)$: 302.2120, found 302.2121.

\subsubsection{6. (Rac-3S,4R,E)- 4-(benzyloxymethyl)-3-propyl-3,4,7,8,9,10-hexahydroazecin-2(1H)-one (18')}

Pentyryl amide 18 (44 mg, $0.14 \mathrm{mmol}$ ) was converted to macrolactam $\mathbf{1 8}^{\prime}$ as an amorphous solid using Procedure A (29 mg, 66\%). IR (KBr) $v_{\max } 3304,2927,2858,1645,1543,1453 \mathrm{~cm}^{-1} ;{ }^{1} \mathrm{H}-\mathrm{NMR}\left(\mathrm{CD}_{3} \mathrm{OD}\right.$, $300 \mathrm{M} \mathrm{Hz}) \delta 7.23-7.15(\mathrm{~m}, 5 \mathrm{H}), 5.42-5.22(\mathrm{~m}, 2 \mathrm{H}), 4.43(\mathrm{~d}, 1 \mathrm{H}, J=12.0 \mathrm{~Hz}), 4.33(\mathrm{~d}, 2 \mathrm{H}, J=12.0 \mathrm{~Hz})$, 3.47-3.43 (m, 2H), $2.63(\mathrm{~m}, 1 \mathrm{H}), 2.30-2.04(\mathrm{~m}, 2 \mathrm{H}), 1.78-0.99(\mathrm{~m}, 10 \mathrm{H}), 0.77(\mathrm{t}, 3 \mathrm{H}, J=6.9 \mathrm{~Hz}) ;{ }^{13} \mathrm{C}-\mathrm{NMR}$ $\left(\mathrm{CDCl}_{3}, 100 \mathrm{M} \mathrm{Hz}\right) \delta 174.8,138.3,133.4,130.3,128.3,127.6$ (2C), 73.1, 69.9, 67.6, 51.5, 47.2, 40.2, 33.0, 30.4, 29.7, 21.4, 14.1; LRMS (FAB) $\mathrm{m} / \mathrm{z} 316\left(\mathrm{M}+\mathrm{H}^{+}\right)$; HRMS (FAB) calcd for $\mathrm{C}_{20} \mathrm{H}_{30} \mathrm{NO}_{2}\left(\mathrm{M}+\mathrm{H}^{+}\right)$: 316.2277, found 316.2272 . 


\subsubsection{2-Ethyl-N-(4-methoxybenzyl)-3-methylpent-4-enamide (21')}

To a solution of crotyl amide 21 (44 mg, $0.18 \mathrm{mmol})$ in toluene $(2 \mathrm{~mL}), i \operatorname{PrMgCl}(2.0 \mathrm{M}$ in THF, $0.18 \mathrm{~mL}, 0.36 \mathrm{mmol}$ ) was added at reflux condition. After stirring at same temperature for $12 \mathrm{~h}$, additional $i \operatorname{PrMgCl}(2.0 \mathrm{M}$ in THF, $0.18 \mathrm{~mL}, 0.36 \mathrm{mmol})$ was added at same temperature. Reaction mixture was refluxed $5 \mathrm{~h}$ and cooled down to room temperature and quenched with brine and extracted with EtOAc. Organic layers were dried over $\mathrm{MgSO}_{4}$, filtered, evaporated, and purified by column chromatography on silica gel (EtOAc: $n$-hexane $=1: 3)$ to afford lactam $21^{\prime}(18 \mathrm{mg}, 36 \%)$ as an amorphous solid. IR (KBr) $v_{\max } 3305,2965,1646,1514,1459 \mathrm{~cm}^{-1} ;{ }^{1} \mathrm{H}-\mathrm{NMR}\left(\mathrm{CDCl}_{3}, 500 \mathrm{M} \mathrm{Hz}\right) \delta 7.18(\mathrm{~d}, 2 \mathrm{H}$, $J=7.6 \mathrm{~Hz}), 6.83(\mathrm{~d}, 2 \mathrm{H}, J=7.6 \mathrm{~Hz}), 5.79-5.72(\mathrm{~m}, 1 \mathrm{H}), 5.59(\mathrm{bs}, 1 \mathrm{H}), 5.00-4.92(\mathrm{~m}, 2 \mathrm{H}), 4.32(\mathrm{~d}, 2 \mathrm{H}, J=$ $14 \mathrm{~Hz}), 3.77(\mathrm{~s}, 3 \mathrm{H}), 2.40-2.34(\mathrm{~m}, 1 \mathrm{H}), 1.83-1.77(\mathrm{~m}, 1 \mathrm{H}), 1.71-1.48(\mathrm{~m}, 3 \mathrm{H}), 1.11(\mathrm{~d}, 3 \mathrm{H}, J=5.5 \mathrm{~Hz}), 0.87$ $(\mathrm{t}, 3 \mathrm{H}, \mathrm{J}=5.9 \mathrm{~Hz}) ;{ }^{13} \mathrm{C}-\mathrm{NMR}\left(\mathrm{CDCl}_{3}, 125 \mathrm{M} \mathrm{Hz}\right) \delta 174.1,158.9,141.6,130.5,129.2,114.2,114.0,55.3,55.2$, 42.8, 40.1, 23.0, 17.5, 12.2; LRMS (FAB) $m / z 262\left(\mathrm{M}+\mathrm{H}^{+}\right)$; HRMS (FAB) calcd for $\mathrm{C}_{16} \mathrm{H}_{24} \mathrm{NO}_{2}\left(\mathrm{M}+\mathrm{H}^{+}\right)$: 262.1807, found 262.1804 .

\subsection{Molecular Dynamics Simulations}

In order to investigate and compare the structural stability of the reaction intermediate of the compounds, MD simulations were performed for $10 \mathrm{~ns}$ with GROMACS 5.1.5 using optimized potentials for liquid simulations-all-atom (OPLS-AA) [19]. The OPLS-AA force field was utilized to explain the interactions that involved lithium. Our ligand topology for the OPLS-AA force field was generated from the LigParGen server [20]. We have modified the parameter file for the oxygen-metal interaction since the original OPLS-AA force field did not provide it. The systems were solvated in the dodecahedron water box with tip3p water model, and then neutralized with the counter ions [21]. Each solvated system was energy minimized through steepest descent algorithm at 10,000 steps and maximum force lower than $1000 \mathrm{~kJ} / \mathrm{mol}$. In two equilibration steps, firstly the equilibration of each system was subjected to number of particles, volume, and temperature (NVT) equilibration at 100 ps at $300 \mathrm{~K}$ using Berendsen thermostat algorithm [22]. Secondly, the equilibration of each system was conducted using number of particles, pressure, and temperature (NPT) equilibration for 100 ps of 1 bar using the Parrinello-Rahman barostat [23]. The Linear Constraint Solver for Molecular Simulations (LINCS) algorithm [24] was used to restrain bond and heavy atom bonds. The settle algorithm [25] was utilized to restrict the geometry of water molecules. We calculated the long-range electrostatic interaction and a cut-off distance of $1.2 \mathrm{~nm}$ using the Particle Mesh Ewald (PME) algorithm [26]. MD simulations were conducted for $10 \mathrm{~ns}$, saving the coordinate data for every 5 ps.

\section{Conclusions}

$i \mathrm{PrMgCl}$ is an advanced base of ACR. In addition, it is superior to the classic LHMDS/toluene condition when the sterically demanded substrate is subjected to ACR. The improvement of the aspect of reactions is derived from the relative stability of the desired conformation for ACR. MD simulations were carried out to investigate the structural stability of the reaction intermediates. The results of C-C-C-O dihedral angle analysis clearly showed that the structural stability of the intermediates was highly related to the portion of the product in the reaction. More detailed mechanistic investigations using other substrates, as well as their applications to the synthesis of bioactive molecules or natural products, will be discussed in due course.

Author Contributions: S.-M.P. conceived the hypothesis, supervised the project, and wrote the manuscript. B.R.S. and M.W.H. performed the synthesis of molecules. D.K. and C.P. performed molecular dynamics studies. K.W.L. supervised the molecular dynamics study and partly wrote the manuscript.

Funding: This research was supported by Basic Science Research Program through the National Research Foundation of Korea (NRF) funded by the ministry of Education, Science, and Technology (NRF-2016R1C1B2006699). Conflicts of Interest: The authors declare no conflict of interest. 


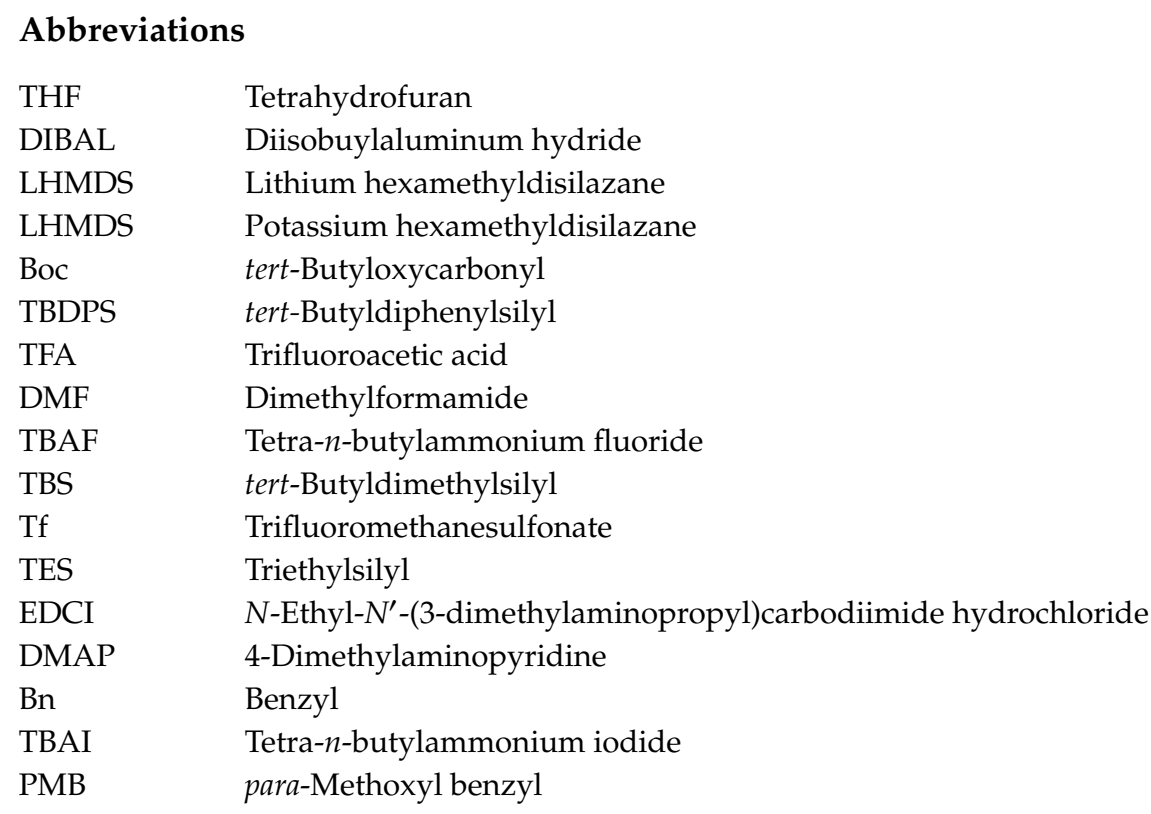

\section{References}

1. Amirkia, V.; Heinrich, M. Alkaloids as Drug leads-A Predictive Structural and Biodiversity-Based Analysis. Phytochem. Lett. 2014, 10, XLVIII-LIII. [CrossRef]

2. Earley, W.G.; Jacobsen, J.E.; Madin, A.; Meier, G.P.; O’Donnell, C.J.; Oh, T.; Old, D.W.; Overman, L.E.; Sharp, M.J. Aza-Cope Rearrangement-Mannich Cyclizations for the Formation of Complex Tricyclic Amines: Stereocontrolled Total Synthesis of ( \pm )-Gelsemine. J. Am. Chem. Soc. 2005, 127, 18046-18053. [CrossRef] [PubMed]

3. Moon, H.; An, H.; Sim, J.; Kim, K.; Paek, S.; Suh, Y. Efficient Strategy for the Stereoselective Synthesis of 2, 3-Disubstituted Benzo [A] Quinolizidine Alkaloids: Concise Synthesis of (-)-Protoemetinol. Tetrahedron Lett. 2015, 56, 608-611. [CrossRef]

4. Kretzschmar, M.; Hofmann, F.; Moock, D.; Schneider, C. Intramolecular Aza-Diels-Alder Reactions of ortho-Quinone Methide Imines: Rapid, Catalytic, and Enantioselective Assembly of Benzannulated Quinolizidines. Angew. Chem. Int. Ed. 2018, 57, 4774-4778. [CrossRef] [PubMed]

5. Crossley, S.W.; Shenvi, R.A. A Longitudinal Study of Alkaloid Synthesis Reveals Functional Group Interconversions as Bad Actors. Chem. Rev. 2015, 115, 9465-9531. [CrossRef]

6. Majumdar, K.C.; Bhattacharyya, T.; Chattopadhyay, B.; Sinha, B. Recent Advances in the Aza-Claisen Rearrangement. Synthesis 2009, 2117-2142. [CrossRef]

7. Martín Castro, A.M. Claisen Rearrangement Over the Past Nine Decades. Chem. Rev. 2004, 104, $2939-3002$. [CrossRef]

8. Lee, Y.; Jung, J.; Kim, S.; Jung, J.; Paek, S.; Kim, N.; Chang, D.; Lee, J.; Suh, Y. First Total Synthesis and Structural Confirmation of Fluvirucinine $\mathrm{A}_{2}$ Via an Iterative Ring Expansion Strategy. Org. Lett. 2010, 12, 2040-2043. [CrossRef]

9. Nubbemeyer, U. Recent advances in charge-accelerated Aza-Claisen rearrangements. In Natural Products Synthesis II; Springer: Berlin, Germany, 2005; pp. 149-213.

10. Jung, J.; Kim, S.; Suh, Y. Advances in Aza-Claisen-Rearrangement-Induced Ring-Expansion Strategies. Asian J. Org. Chem. 2017, 6, 1117-1129. [CrossRef]

11. Tsunoda, T.; Sasaki, O.; Itô, S. Aza-Claisen Rearrangement of Amide Enolates. Stereoselective Synthesis of 2, 3-Disubstituted Carboxamides. Tetrahedron Lett. 1990, 31, 727-730. [CrossRef]

12. Suh, Y.; Kim, S.; Jung, J.; Shin, D.; Min, K.; Koo, B.; Kim, H. Asymmetric Total Synthesis of Fluvirucinine $A_{1}$. Angew. Chem. Int. Ed. 1999, 38, 3545-3547. [CrossRef]

13. Paek, S.; Kim, N.; Shin, D.; Jung, J.; Jung, J.; Chang, D.; Moon, H.; Suh, Y. A Concise Total Synthesis of (+)-Tetrabenazine and (+)- $\alpha$-Dihydrotetrabenazine. Chem. Eur. J. 2010, 16, 4623-4628. [CrossRef] [PubMed]

14. Sonntag, N.O. The Reactions of Aliphatic Acid Chlorides. Chem. Rev. 1953, 52, 237-416. [CrossRef] 
15. Moon, H.; Yoon, H.; Lim, C.; Jang, J.; Yi, J.; Lee, J.; Lee, J.; Na, Y.; Son, W.; Kim, S. Asymmetric Synthesis of (-)-6-Desmethyl-Fluvirucinine A1 Via Conformationally-Controlled Diastereoselective Lactam-Ring Expansions. Molecules 2018, 23, 2351. [CrossRef] [PubMed]

16. Jang, J.; Jung, J.; Ahn, J.; Sim, J.; Chang, D.; Kim, D.; Suh, Y. Asymmetric Formal Synthesis of Schulzeines A and C. Org. Biomol. Chem. 2012, 10, 5202-5204. [CrossRef]

17. Arnold, R.T.; Searles, S. A New Rearrangement of Allylic Esters. J. Am. Chem. Soc. 1949, 71, 1150-1151. [CrossRef]

18. Sato, T.; Yamazaki, T.; Nakanishi, Y.; Uenishi, J.; Ikeda, M. A New Entry to 9-Azabicyclo [3.3. 1] Nonanes using Radical Translocation/Cyclisation Reactions of 2-(but-3-Ynyl)-1-(O-Iodobenzoyl) Piperidines. J. Chem. Soc. Perkin Trans. 1 2002, 1438-1443. [CrossRef]

19. Abraham, M.J.; Murtola, T.; Schulz, R.; Páll, S.; Smith, J.C.; Hess, B.; Lindahl, E. GROMACS: High Performance Molecular Simulations through Multi-Level Parallelism from Laptops to Supercomputers. SoftwareX 2015, 1, 19-25. [CrossRef]

20. Dodda, L.S.; Cabeza de Vaca, I.; Tirado-Rives, J.; Jorgensen, W.L. LigParGen Web Server: An Automatic OPLS-AA Parameter Generator for Organic Ligands. Nucleic Acids Res. 2017, 45, W331-W336. [CrossRef]

21. Jorgensen, W.L.; Chandrasekhar, J.; Madura, J.D.; Impey, R.W.; Klein, M.L. Comparison of Simple Potential Functions for Simulating Liquid Water. J. Chem. Phys. 1983, 79, 926-935. [CrossRef]

22. Berendsen, H.J.; Postma, J.V.; van Gunsteren, W.F.; DiNola, A.; Haak, J.R. Molecular Dynamics with Coupling to an External Bath. J. Chem. Phys. 1984, 81, 3684-3690. [CrossRef]

23. Parrinello, M.; Rahman, A. Polymorphic Transitions in Single Crystals: A New Molecular Dynamics Method. J. Appl. Phys. 1981, 52, 7182-7190. [CrossRef]

24. Hess, B.; Bekker, H.; Berendsen, H.J.; Fraaije, J.G. LINCS: A Linear Constraint Solver for Molecular Simulations. J. Comput. Chem. 1997, 18, 1463-1472. [CrossRef]

25. Miyamoto, S.; Kollman, P.A. Settle: An Analytical Version of the SHAKE and RATTLE Algorithm for Rigid Water Models. J. Comput. Chem. 1992, 13, 952-962. [CrossRef]

26. Darden, T.; York, D.; Pedersen, L. Particle Mesh Ewald: An N Log (N) Method for Ewald Sums in Large Systems. J. Chem. Phys. 1993, 98, 10089-10092. [CrossRef]

Sample Availability: Samples of the compounds 1-21' are available from the authors.

(C) 2019 by the authors. Licensee MDPI, Basel, Switzerland. This article is an open access article distributed under the terms and conditions of the Creative Commons Attribution (CC BY) license (http://creativecommons.org/licenses/by/4.0/). 